\title{
RINGS OF CONTINUOUS INTEGER-VALUED FUNCTIONS AND NONSTANDARD ARITHMETIC $\left({ }^{1}\right)$
}

\author{
BY \\ NORMAN L. ALLING
}

0. Introduction. In this paper rings of continuous integer-valued functions are studied, with particular attention paid to their maximal residue class domains. These domains correspond bijectively to minimal prime ideals, rendering the space of these ideals of particular interest. Since these domains are either the integers or are nonstandard models of the integers, questions about nonstandard arithmetic will also be considered.

In $\$ 1$ the space of minimal prime ideals of $C(X, Z)$, the ring of continuous functions from a nonempty Hausdorff space $X$ into $Z$, the ring of integers, is showed to be homeomorphic to $\delta X(1.2)$, the Boolean space of the algebra of open-and-closed sets of $X$. The maximal ideal space of $C(X, Z)$ is shown to map continuously onto $\delta X$ (1.3). The space, $\delta_{0} X$, of points of $\delta X$ that give rise to integer residue class domains, is studied in $\S 2$. The map of $X$ into $\delta_{0} X$ strongly resembles the realcompactification injection [GJ]. A representation theorem of $C(X, Z)$ over $\delta_{0} X$ is also given (2.4).

It is shown in $\S 3$ that points in $\delta X-\delta_{0} X$ give rise to $Z$, a nonstandard model of $Z$ (3.1). Here some of the relevant background material in model theory is discussed. The algebraic theory of nonstandard arithmetic is studied in $\S 4$. In $\S 5$ we return to study $Z$, its maximal ideal space, and its quotient field $Q$, which is a nonstandard model of the rational field $Q$. In $\S 6$, the most technical section of the paper, the valuations of $\boldsymbol{Q}$ associated with maximal ideals of $\boldsymbol{Z}$ are computed (6.3). The value groups that arise are analysed ((6.4), (6.5), and (6.6)), followed by some rather striking results in case the maximal ideal in question is principal.

The ideals of $\boldsymbol{Z}$ are analyzed in $\$ 7$ along classical lines: i.e., we proceed from the study of maximal and prime ideals, through the study of primary ideals, to a decomposition theorem for ideals in terms of primary ideals (7.4). Ideals in $C(X, Z)$ are decomposed in $\S 8$, first into coprimary ideals (8.4), and then into primary ideals (8.9). In the process, the sets of maximal, prime, coprimary, and primary ideals of $C(X, Z)$ are analyzed.

In $\$ 9$ some model-theoretic results are obtained on the residue class fields of $C(X, Z)$, the principal result being that any such field is elementarily equivalent

Presented to the Society, January 23, 1964 under the title Rings of integer-valued functions and nonstandard models; received by the editors June 12, 1964.

(1) This research wassupported in part by National Science Foundation grant NSF-GP-379. 
to some residue class field of any fixed nonstandard $Z$ (9.7). In addition, two questions raised by Pierce are settled [P, 7.2 and 7.3].

The paper is built on the foundations laid down by Pierce in Rings of integervalued continuous functions $[\mathrm{P}]$.

1. Minimal prime ideals in $C(X, Z)$. Let $\mathfrak{M}$ be the space of maximal ideals of $C(X, Z)$ under the Stone topology [GJ, 7M]. (Note: whenever we speak of the maximal (prime) ideal space of a commutative ring with identity, it will be understood to have the Stone (Zariski) topology.) $\mathfrak{M}$ is a compact $T_{1}$-space $[\mathrm{P}, 3.1]$. Let $\Pi$ be the set of (positive) prime numbers. Given $f \in C(X, Z)$, let $D(f)=\{(x, p) \in X \times \Pi: f(x) \equiv 0 \bmod p\} . D$ has the following properties $[\mathrm{P}, 2.2 .1]$ : $D(f) \cup D(g)=D(f g), D(f) \cap D(g) \subset D(f-g)$, and $D(f) \cap D(g)=D((f, g))$, where $(f, g)$ denotes the ideal $(d)$ generated by $f$ and $g$ in $C(X, Z)[\mathrm{P}, 1.3 .2]$. Clearly, $D$ induces a mapping on subsets of $C(X, Z)$ into subsets of $X \times \Pi$; let this mapping also be denoted by $D$. Let $\mathscr{D}=D C(X, Z)$ and let $\mathfrak{D}$ be the space of all ultrafilters of $\mathscr{D}$, under the hull-kernel topology [P, 3]. (Note: whenever we speak of a space of ultrafilters it will be understood to have the hull-kernel topology.) $D$ is a homeomorphism of $\mathfrak{M}$ onto $\mathfrak{D}[P, 3.1 .2]$.

Let $\xi$ be the first projection of $X \times \Pi$. $\xi$ induces a mapping of $\mathscr{D}$ onto $\mathscr{B}$, the open-and-closed subsets of $X$, which we will call $\xi$.

Let $\delta X$ be the space of ultrafilters of $\mathscr{B}$. Then $\delta X$ is a totally disconnected Hausdorff space $[\mathrm{P}, 1.6]$. Let $\xi$ be the mapping of $\mathfrak{D}$ into $\delta X$ induced by $\xi$. It is easy to see that $\xi$ maps $\mathfrak{D}$ onto $\delta X$. Then $\mathscr{U} \equiv \xi D$ maps $\mathfrak{M}$ onto $\delta X$.

Given $f \in C(X, Z)$, let $\zeta(f)=\{x \in X: f(x)=0\}$. $\zeta$ maps $C(X, Z)$ onto $\mathscr{B}[\mathrm{P}, 1.2]$, and has the following properties: $\zeta(f) \cup \zeta(g)=\zeta(f g), \zeta(f) \cap \zeta(g) \subset \zeta(f-g)$, and $\zeta(f) \cap \zeta(g)=\zeta((f, g))=\zeta\left(f^{2}+g^{2}\right)$. (Cf. [GJ, 1.10].) $\zeta$ induces a map $\zeta$ of subsets of $C(X, Z)$ into subsets of $\mathscr{B}$. For $\mu \in \delta X$, let $\zeta^{-1}(\mu) \equiv P_{\mu}=\{f \in C(X, Z)$ : $\zeta(f) \in \mu\}$. It is easily seen that $P_{\mu}$ is a prime ideal of $C(X, Z)$ [GJ, 2.3 and 2.12].

Theorem 1.1. Let $M \in \mathfrak{M}, \mu=\mathscr{U}(M)$, and let $P_{\mu}=\zeta^{-1}(\mu)$. $P_{\mu}$ is the unique minimal prime ideal of $C(X, Z)$ that is contained in $M$.

Proof. Let $P$ be a prime ideal of $C(X, Z)$ that is contained in $M$, let $f \in P_{\mu}$, and let $\zeta(f)=U$. Since $P_{\mu}=\zeta^{-1}(\mu), U$ is in $\mu$. As a result, $g=\chi_{U}$, the characteristic function of $U$ on $X$, is in $C(X, Z)$. Since $\mathscr{U}(g)=X-U$ is not in $\mu$, $g \notin M$. By hypothesis, $P \subset M$, therefore, $g \notin P$; but $f g=0 \in P$. We conclude that $f \in P$, showing that $P_{\mu} \subset P$, proving the theorem. (Cf. [GJ, 7.15].)

Let $\mathfrak{P}_{0}$ be the space of minimal prime ideals of $C(X, Z)$. By $(1.1), \zeta^{-1}$ is a mapping of $\delta X$ onto $\mathfrak{P}_{0}$. Clearly, $\zeta\left(P_{\mu}\right) \equiv\left\{\zeta(f): f \in P_{\mu}\right\}=\mu$, showing that $\zeta^{-1}$ is injective. Since $\zeta^{-1}$ preserves intersection, we have the following. (Cf. [P, 3.1.2].)

PROPOSITION 1.2. $\zeta$ is a homeomorphism of $\mathfrak{P}_{0}$ onto $\delta X$.

REMARK. Let $C(X, Q)$ denote the ring of continuous maps of $X$ into $Q$, the 
rationals, under the discrete topology. Given $f \in C(X, Q)$ let $\zeta^{\prime}(f)=\{x \in X: f(x)=0\}$, and let $\mathfrak{M}^{\prime}$ be the space of maximal ideals of $C(X, Q) . \zeta^{\prime}$ is then a homeomorphism of $\mathfrak{M}^{\prime}$ onto $\delta X$ (cf. [GJ, 7.11]). Given $M^{\prime} \in \mathfrak{M}^{\prime}, M^{\prime} \cap C(X, Z)=P_{\mu}$, where $\mu=\zeta^{\prime}\left(M^{\prime}\right)$. Thus $M^{\prime} \rightarrow M^{\prime} \cap C(X, Z)$ is homeomorphism of $\mathfrak{M}^{\prime}$ onto $\mathfrak{P}_{0}$ that commutes with the mappings $\zeta^{\prime}$ and $\zeta^{-1}$.

Let $\sigma \equiv \zeta^{-1} \xi D$, and consider the following commutative diagram.

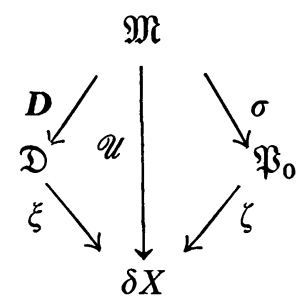

Since $D, \xi$, and $\zeta^{-1}$ are surjective, so is $\sigma$.

THEOREM 1.3. $\sigma$ is a continuous mapping of $\mathfrak{M}$ onto $\mathfrak{P}_{0}$ that takes a maximal ideal to the unique minimal prime ideal contained in it. Since $\boldsymbol{D}$ and $\zeta$ are surjective homeomorphisms, $\xi$ and $\mathscr{U}$ are continuous.

Proof. Given $f \in C(X, Z)$, let $\mathfrak{P}_{0}(f) \equiv\left\{P \in \mathfrak{P}_{0}: f \in P\right\}$. The set of all sets of this form constitutes a base of the closed sets of $\mathfrak{P}_{0}$. Let $g=|f| \vee 1$, and note that $\mathfrak{P}_{0}(g)=\mathfrak{P}_{0}(f)$. Clearly $\sigma^{-1} \mathfrak{P}_{0}(g) \subset \mathfrak{M}(g) \equiv\{M \in \mathfrak{M}: g \in M\}$. We will show that these two sets are equal, proving that $\sigma$ is continuous. By construction, $D(g)=\zeta(g) \times \Pi$; thus $M \in \mathfrak{M}(g)$ implies $\zeta(g) \in \mathscr{U}(M)=\zeta \sigma(M)$, proving that $\bullet(M) \in \mathfrak{P}_{0}(g)$, Therefore, $\mathfrak{M}(g) \subset \sigma^{-1} \mathfrak{P}_{0}(g)$, proving the theorem.

Proposition 1.4. Let $f \in C(X, Z)$. Then $\mathfrak{P}_{0}(f)$ is an open-and-closed subset of $\mathfrak{P}_{0}$.

Proof. Let $\zeta(f)=U$ and let $V=X-U$. $U$ and $V$ are in $\mathscr{B}$ and partition $X$. Let $\theta$ be the natural injection of $X$ into $\delta X$. Since $\delta X$ is the space of ultrafilters of $\mathscr{B}, \mathrm{cl}_{\delta X} \theta U$ and $\mathrm{cl}_{\delta X} \theta V$ partition $\delta X$. Since $\delta^{-1} \operatorname{cl}_{\delta X} \theta U=\mathfrak{P}_{0}(f)$, the proposition is proved.

2. Maximal residue class domains of $C(X, Z)$, standard theory. Maximal residue class domains of $C(X, Z)$ correspond bijectively with minimal prime ideals of $C(X, Z)$. Let $P \in \mathfrak{P}_{0}$ and let $Z \equiv C(X, Z) / P$. Given $f \in C(X, Z)$, let $f$ be its image in $Z$. Conversely, given $f \in Z$, let $f$ be a pre-image of $f$ in $C(X, Z)$. Given a subset $S$ of $Z$ let $S$ be the pre-image of $S$ in $C(X, Z)$ and given $S$ in $C(X, Z)$ let $S$ be its image in $Z$.

Proposition 2.1. $Z$ has a unique total ordering under which $f \rightarrow f$ is a latticehomomorphism. The subring of $Z$ generated by 1 , called $Z$, is the smallest nonzero convex subring of $\boldsymbol{Z}$. 
Proof. Given $f \in P$ and $g \in C(X, Z)$ such that $|g| \leqq|f|$; then $\zeta(f) \subset \zeta(g)$. Since $P=\zeta^{-1}(\mu)$ for some $\mu \in \delta X$ and since $\mu$ is a filter, $g \in P$. This shows that the natural order on $C(X, Z)$ induces a unique lattice ordering on $Z$ under which $f \rightarrow \boldsymbol{f}$ is a lattice-homomorphism [GJ, 5.3]. To see that $\boldsymbol{Z}$ is totally ordered under this ordering, let $f \in C(X, Z)$, let $U=\{x \in X: f(x) \geqq 0\}$, and let $V=\{x \in X: f(x)<0\} . U$ and $V$ are in $\mathscr{B}$ and partition $X$. Since $\mu$ is an ultrafilter in $\mathscr{B}$, either $U$ or $V$ is in $\mu$. Accordingly, either $f \equiv f \chi_{U}(\bmod P)$ or $f \equiv f \chi_{V}$ $(\bmod P)$. Since $f \chi_{U} \geqq 0$ and $f \chi_{V}<0$, the proposition is proved. (Cf. [GJ, 5.5].)

Let $\mathrm{m}$ be the space of maximal ideals of $Z . m$ is a compact $T_{1}$ space that is not Hausdorff [GJ, 7M]. The inverse of $f \rightarrow f$ induces an injection $M \rightarrow M$ of $\mathrm{m}$ onto $\sigma^{-1}(P)$, the fibre of $\mathfrak{M}$ over $P$.

Proposition 2.2. The injection $M \rightarrow M$ of $\mathrm{m}$ onto $\sigma^{-1}(P)$ is a homeomorphism.

Proof. Sets of the form $\mathfrak{m}(\boldsymbol{f})=\{\boldsymbol{M} \in \mathfrak{m}: \boldsymbol{f} \in \boldsymbol{M}\}$, for $\boldsymbol{f} \in \boldsymbol{Z}$, form a base for the closed sets in $m$. It suffices to show that given $f \in C(X, Z)$, the image of $m(f)$ in $\sigma^{-1}(P)$ is $\mathfrak{M}(f) \cap \sigma^{-1}(P)$; but this is immediate, proving the proposition.

$X$ is said to be $Z$-pseudocompact if $C(X, Z)=C^{*}(X, Z)$, the set of bounded continuous maps of $X$ into $Z[P, 1.8 .1]$.

Proposition 2.3. $X$ is $Z$-pseudocompact if and only if $Z=Z$ for all $P \in \mathfrak{P}_{0}$.

Proof. If $X$ is $Z$-pseudocompact, then $Z$ is cofinal in $Z$ for all $P \in \mathfrak{P}_{0}$. Conversely, assume that $X$ is not $Z$-pseudocompact. There exist $f \in C(X, Z)-C^{*}(X, Z)$. Let $U_{n}=\{x \in X:|f(x)| \geqq n\}$. Clearly $\left(U_{n}\right)_{n \in N}$ is a family of subsets of $\mathscr{B}$ having the finite intersection property. By Zorn's Lemma, $\left(U_{n}\right)_{n \in N}$ is contained in some $\mu \in \delta X$. Let $P=\zeta^{-1}(\mu)$ and let $Z=C(X, Z) / P$. Then $|f| \geqq n$ for all $n \in N$, proving the proposition. (Cf. [GJ, 8.4].)

Given $x \in X$ let $\theta(x)$ be the set of all subsets of $\mathscr{B}$ that contain $x . \theta$ is a continuous mapping of $X$ onto a dense subset of $\delta X[\mathrm{P}, 1.5 .2]$. Let $\delta_{0} X=\left\{\mu \in \delta X: C(X, Z) / P_{\mu}=Z\right\}$. Then $\theta X \subset \delta_{0} X \subset \delta X$ and by (2.3) $\delta_{0} X=\delta X$ if and only if $X$ is $Z$-pseudocompact.

THeOREM 2.4. Every $f \in C(X, Z)$ extends uniquely, by duality, to $\hat{f} \in C\left(\delta_{0} X, Z\right)$. The map $f \rightarrow \hat{f}$ is a surjective lattice-isomorphism. $\delta_{0} X=\{\mu \in \delta X: \mu$ has the countable intersection property $\}. \delta_{0} X$ is the largest space (up to homeomorphism) in which $\theta X$ is dense, such that each $f \in C(X, Z)$ extends to $\hat{f} \in C\left(\delta_{0} X, Z\right)$, the functions $\{\hat{f}: f \in C(X, Z)\}$ separating the points of $\delta_{0} X$.

Since this sort of theorem is by now familiar, its proof will only be sketched. (Cf. [GJ, Chapter 8].)

By duality, $\hat{f} \in Z^{\delta_{0} X}$. Clearly, $f \rightarrow \hat{f}$ is a lattice-isomorphism. To show that $\hat{f}$ is continuous, it suffices to show that, given any $n \in Z, \hat{f}^{-1}(n)$ is open-and-closed in $\delta_{0} X . \theta^{*}$, the adjoint of $\theta$, is a lattice-isomorphism of $C^{*}(\delta X, Z)$ onto $C^{*}(X, Z)$ $[\mathrm{P}, 1.5 .2]$. Given $h \in C^{*}(X, Z)$, let $h^{\delta}=\left(\theta^{*}\right)^{-1}(h)$. Clearly, $h^{\delta} \mid \delta_{0} X=\hat{h}$. Let 
$g=(|n|+1) \wedge f \vee(-|n|-1)$. Since $g \in C^{*}(X, Z), \quad(g) \quad(n) \equiv U$ is an open-and-closed subset of $\delta X$. Therefore $U \cap \delta_{0} X$ is an open-and-closed subset of $\delta_{0} X$, but this set is $\hat{f}^{-1}(n)$, showing that $\hat{f}$ is continuous. By [P, 1.2], $f \rightarrow \hat{f}$ is surjective.

To show that $\delta_{0} X=\{\mu \in \delta X: \mu$ has the countable intersection property $\}$ (see [GJ, 0.3] for definition), it suffices to show that $\delta X-\delta_{0} X$ is $\{\mu \in \delta X: \mu$ does not have the countable intersection property $\}$. Given such $\mu$, let $\left(U_{n}\right)_{n \in N}$ be a subset of $\mu$ having an empty intersection. Let $f=\Sigma_{n \in N} \chi_{U_{n}}$. Clearly, $f \in C(X, Z)$ and $f \in C(X, Z) / P_{\mu}-Z$. Conversely, if $\mu \in \delta X-\delta_{0} X$, there exists $f \in C(X, Z) / P_{\mu}-Z$. Let $U_{n}=\{x \in X:|f| \geqq n\}$. Each $U_{n}$ is in $\mu$ and $\bigcap_{n \in N} U_{n}=\varnothing$.

To prove the last assertion, let $\phi$ be a homeomorphism of $\delta_{0} X$ onto a dense subset of a Hausdorff space $Y$ such that, for each $\hat{f} \in C\left(\delta_{0} X, Z\right)$, there exists $\tilde{f} \in C(Y, Z)$ such that $\hat{f}=\tilde{f} \phi$, the maps $\tilde{f}$ separating the points of $Y$. $\phi^{*}$ is a latticeisomorphism of $C(Y, Z)$ onto $C\left(\delta_{0} X, Z\right)$. Further, $f \rightarrow \hat{f} \rightarrow \hat{f}$ defines surjective isomorphisms of the following rings: $C(X, Z) \rightarrow C\left(\delta_{0} X, Z\right) \rightarrow C(Y, Z)$. A point $y \in Y$ gives rise, by means of evaluation, to a homomorphism of $C(Y, Z)$ onto $Z$, and thus the kernel $P$ of this mapping pulled back to $C(X, Z)$ is in $\zeta^{-1}\left(\delta_{0} X\right)$. Let $\mu=\zeta(P)$, and note that the homomorphism pulled back to $C\left(\delta_{0} X, Z\right)$ is $\hat{f} \rightarrow \hat{f}(\mu)$. We conclude that $\tilde{f}(y)=\tilde{f}(\phi(\mu))$ for all $\tilde{f} \in C(Y, Z)$. By hypothesis, these functions $\tilde{f}$ separate the points of $Y$, showing that $\phi(\mu)=y$, and that $\phi$ is a homeomorphism of $\delta_{0} X$ onto $Y$, concluding our sketch of the proof of (2.4).

EXAMPLE. Let $W$ be the space of ordinals less than the first uncountable ordinal, under the order topology. Then $\theta W \neq \delta_{0} W$. (See [GJ, 5.12] for details.)

Historical note. Many of the results of this section are similar to results obtained by Hewitt $\left[\mathrm{H}_{2}\right]$ for real-valued functions.

3. Maximal residue class domains of $C(X, Z)$, nonstandard theory. Unless otherwise stated, assume henceforth that $X$ is not $Z$-pseudocompact. We have seen (2.3) that there exist $\mu \in \delta X-\delta_{0} X$, and that $Z \equiv C(X, Z) / P_{\mu}$ is a proper extension of $Z$.

\section{THEOREM 3.1. $Z$ is a nonstandard model of $Z$.}

Let us recall some definition from model theory, in order to clarify the meaning of this theorem.

Let $\mathfrak{A}$ be the category of all totally ordered integral domains and order-preserving homomorphisms. For such domains, equality, addition, multiplication, and order are the atomic relations [R, Chapter II]. The lower predicate calculus as applied to objects in $\mathfrak{A}, L(\mathfrak{H})$, is built up by means of certain rules from atomic relations, object symbols, and variables; the logical connectives of negation, disjunction, conjunction, implication, and equivalence; and the universal and existential quantifiers, together with brackets [R, 1.2]. Let $\phi \in L(\mathfrak{U})$, and let $x_{1}, \cdots, x_{n}, \cdots$ be its free variables $[\mathrm{R}, \mathrm{p} .5]$. Let $a_{1}, \cdots, a_{n}, \cdots$ be elements in an 
object $A$ in $\mathfrak{A}$, and let $(a)$ be the vector $\left(a_{1}, \cdots, a_{n}, \cdots\right)$. (a) is said to satisfy $\phi$ if, when $a_{n}$ is substituted for $x_{n}$ in $\phi$, the resulting statement $\phi(a)$ holds in $A$. For example, if $\phi$ is equivalent to the mathematical statement, $x_{1}=x_{2}$, then $\phi(a)$ holds in $A$ if and only if $a_{1}=a_{2} . \phi$ is said to be valid in $A$ if $\phi(a)$ holds in $A$ for all (a). For example, if $\phi$ is equivalent to the mathematical statement, $x_{1}+x_{2}=x_{2}+x_{1}$, then $\phi$ is valid in $A$. A sentence in $L(\mathfrak{U})$ is an element in $L(\mathfrak{A})$ without free variables. By an elementary theorem about $A$ is meant a theorem concerning $A$ that can be stated as a sentence in $L(\mathfrak{A})$. Two objects in $\mathfrak{U}$ are said to be elementarily equivalent, if every elementary theorem valid in one is valid in the other. Clearly isomorphic objects are elementarily equivalent. However, elementarily equivalent objects in $\mathfrak{U}$ need not be isomorphic. An object in $\mathfrak{U}$ that is elementarily equivalent to $Z$ but is not isomorphic to it will be called a nonstandard model of $Z$; thus the meaning of (3.1) is clear.

$\boldsymbol{Z}$ can be written as an inductive limit as follows (see, e.g., [ES, Chapter VIII, §4] for definition): given $U \in \mu$, the restriction mapping of $C(X, Z)$ to $C(U, Z)$ is a lattice-homomorphism (which, in this case, is surjective $[P, 1.2])$. The kernel of the canonical homomorphism of $C(X, Z)$ onto ind $\lim _{U \in \mu} C(U, Z)$ is $P_{\mu}$; thus this inductive limit may be identified with $Z$. If $X$ is discrete we may apply [K, 5.1] to prove (3.1). We will now refine the proof of $[\mathrm{K}, 5.1]$ a bit to cover the nondiscrete case. Generalizing the setting slightly, let $A$ be a countable object in $\mathfrak{U}$ and let $A$ have the discrete topology. For $f \in C(X, A)$ let $\zeta(f)=\{x \in X: f(x)=0\}$. Then $\zeta(C(X, A))=\mathscr{B}$. Finally, let $A=$ ind $\lim _{U \in \mu} C(U, A)$.

Lemma 3.2. Let $\phi \in L(\mathfrak{U})$, let $f_{n} \in C(X, A)$, and let $f_{n}$ be the image of $f_{n}$ in $A$ for all $n \in N$. Let $(f)=\left(f_{1}, \cdots, f_{n}, \cdots\right)$, let $(f)=\left(f_{1}, \cdots, f_{n}, \cdots\right)$, and let $U=\{x \in X: \phi(f(x))$ holds in $A\}$. Then $U$ is in $\mathscr{B}$. Further, $\phi(f)$ holds in $\boldsymbol{A}$ if and only if $U \in \mu$.

Proof. Let $\phi$ and $\psi \in L(\mathfrak{U})$, let $U=\{x \in X: \phi(f(x))$ holds in $A\}$, and let $V=\{x \in X: \psi(f(x))$ holds in $A\}$. By the definition of equality, addition, multiplication, and order in ind $\lim _{U \in \mu} C(U, A)$, the theorem is true if $\phi$ is atomic.

Assume that the lemma holds for $\phi$ and for $\psi$. The set, $\{x \in X: \phi(f(x)) \wedge \psi(f(x))$ holds in $A\}$, is $U \cap V$. Since $\mathscr{B}$ is closed under finite intersection, $U \cap V$ is in $\mathscr{B}$. If $\phi(f) \wedge \psi(f)$ holds in $\boldsymbol{A}, \phi(f)$ holds in $\boldsymbol{A}$ and $\psi(f)$ holds in $\boldsymbol{A}$; thus, by hypothesis, $U, V \in \mu$. Since $\mu$ is a filter, $U \cap V \in \mu$. Conversely, assume that $U \cap V \in \mu$. Since $\mu$ is a filter in $\mathscr{B}$ and since $U$ and $V$ are in $\mathscr{B}, U$ and $V$ are in $\mu$. By hypothesis, $\phi(f)$ and $\psi(f)$ hold in $\boldsymbol{A}$, proving that $\phi(f) \wedge \psi(f)$ holds in $\boldsymbol{A}$.

Assume that the lemma is true for $\phi$, and let $\psi=(\sim \phi)$. The statement, $(\sim \phi)(f)$ holds in $A$, is equivalent to $U \notin \mu$. Since $\mathscr{B}$, the algebra of open-and-closed sets in $X$, is closed under complementation, $V=X-U$ is in $\mathscr{B}$. Since $\mu$ is an ultrafilter in $\mathscr{B}, V \in \mu$. (Cf. [GJ, 2.13].) Conversely, assume that $V \in \mu$. Since $\varnothing \notin \mu, U \notin \mu$, proving that $(\sim \phi)(f)$ is valid in $A$. 
Let $\psi=\left(\exists x_{j}\right) \phi$. First we will show that $V \in \mathscr{B}$. (This is the first point at which the proof in the nondiscrete case is more involved than the proof in the discrete case. Cf. [K, p. 227].) By definition, for each $x \in V$ there exists $t \in A$ such that

$$
\phi\left(f_{1}(x), \cdots, f_{j-1}(x), t, f_{j+1}(x), \cdots\right) \text { holds in } A .
$$

Let $T$ be the set of all such $t \in A$. For each $t \in T$ let $X_{t}$ be the set of all $x \in X$ for which (*) is true. Since the constant function of value $t$ is in $C(X, A)$, we can invoke the induction hypothesis and conclude that $X_{t} \in \mathscr{B}$. Clearly $\bigcup_{t \in T} X_{t}=V$. Since $A$ is, by hypothesis, countable, $T$ is countable and can be properly indexed as follows: $T=(t(n))_{n \in N^{\prime}}$, where $N^{\prime}$ is an initial segment of $N$. Let $V_{1}=X_{t(1)}, V_{2}=X_{t(2)}-V_{1}$, and let $V_{3}=X_{t(3)}-V_{1} \cup V_{2}$; continuing in this fashion, by finite induction, defines a partition $\left(V_{n}\right)_{n \in N^{\prime}}$ of $V$ by elements of $\mathscr{B}$. Let $g \mid V_{n}=t(n)$ and $g \mid X-V$ $=0$; then $g \in C(X, A)$ (cf. [P, 1.2]). Let $\left(f^{\prime}\right)=\left(f_{1}, \cdots, f_{j-1}, g, f_{j+1}, \cdots\right)$ and let $W=\left\{x \in X: \phi\left(f^{\prime}(x)\right)\right.$ holds in $\left.A\right\}$. By hypothesis $W \in \mathscr{B}$, and by the construction of $g, W=V$, showing that $V \in \mathscr{B}$. Assume now that $V \in \mu$. By hypothesis, $\phi\left(\boldsymbol{f}^{\prime}\right)$ holds in $\boldsymbol{A}$ : i.e., $\psi(\boldsymbol{f})$ holds in $\boldsymbol{A}$. Conversely, assume that $\psi(\boldsymbol{f})$ holds in $\boldsymbol{A}$. Then there exists $h \in C(X, A)$ such that if $\left(f^{\prime \prime}\right)=\left(f_{1}, \cdots, f_{j-1}, h, f_{j+1}, \cdots\right)$, then $\phi\left(f^{\prime \prime}\right)$ holds in $A$. Let $Y=\left\{x \in X: \phi\left(f^{\prime \prime}(x)\right)\right.$ holds in $\left.A\right\}$ and note that $Y \subset V$. It was shown above that $V \in \mathscr{B}$. By hypothesis, $Y \in \mu$, therefore $V \in \mu$. Since all formulas in $L(\mathfrak{R})$ can be built up from atomic formulas in a finite number of steps of the kind treated above, the lemma is proved.

Applying (3.2) to sentences proves (3.1).

Historical note. Scott $[\mathrm{S}]$ has considered this method of constructing nonstandard models of $Z$, in case $X$ is discrete.

4. The algebraic theory of nonstandard models of $Z$. Let $A$ be an object in $\mathfrak{A}$. It is easily seen that the following notions about $A$ can be formulated in $L(\mathfrak{H})$ : $a$ is a divisor of $b$ in $A, a$ is a unit in $A, d$ is a greatest common divisor of $a_{1}, \cdots, a_{n}$ in $A, p$ is a prime element of $A$, and $a \equiv b(\bmod c)$ in $A$. Throughout the remainder of this section, let $A$ be a nonstandard model of $Z$.

THEOREM 4.1. The following hold in A:

1. The group of units in $A$ is $\{1,-1\}$.

2. Every finitely generated ideal is principal.

3. (EUCLID's FIRST THEOREM). If $p$ is a prime in $A$ and $p \mid a b$, then $p \mid a$ or $p \mid b$.

4. Every nonunit has a prime factor.

5. Every element of $A$ that can be written as a (finite) product of primes and units is uniquely expressible in this fashion, except for a unit factor and the order of the prime factors.

6. Given a prime $p$ in $A,(p)$ is a maximal ideal.

7. The intersection of all principal maximal ideals in $A$ is the zero ideal: i.e., the set of principal maximal ideals is dense in the space of maximal ideals of $A$. 
8. (Fermat's THEOREM). Given a finite prime $p$ of $A$ and $a \in A-(p)$, then $a^{p-1} \equiv 1(\bmod p)$.

9. (LAGRANGE'S THEOREM). Every non-negative element of $A$ is the sum of four squares.

Proof. Since $A$ is elementarily equivalent to $Z$, and all of the theorems hold in $Z$, it suffices to show that each of the theorems is elementary. Since the notion of a unit in $A$ is expressible in a sentence in $L(\mathfrak{U}), 1$ holds. 2 is equivalent to the statement that a finite number of elements in $A$ has a greatest common divisor, an elementary statement. Since the notion of a prime in $A$ is elementary, 3 is elementary. Since the notion of a prime and of a nonunit are elementary concepts, 4 and 5 hold. 6 is equivalent to the following elementary statement: for all primes $p$ in $A$ and for all $a \in A-(p),(a, p)=1.7$ is equivalent to the following elementary statement: for all nonzero $a$ in $A$, there exists a prime $p$ in $A$ such that $p \nmid a$. Clearly 8 and 9 are elementary statements. Thus the theorem has been proved.

ExAmple. Let $X=N$, let $\mu \in \delta X-\delta_{0} X$, let $f(n)=n !$; and let $Z=$ ind $_{\lim _{U \in \mu}} C(U, Z)$. Every finite prime in $Z$ divides $f$, but $f \neq 0$; thus the finite principal maximal ideals of $\boldsymbol{Z}$ are not dense in the maximal ideal space of $\boldsymbol{Z}$. Using (4.1.7) we see that there must exist infinite primes in $Z$. Further, in spite of (4.1.5), the unique factorization theorem does not hold in $Z$.

COROllary 4.2. If $p$ is a finite prime in $A$, then $A /(p)=Z /(p)$.

Proof. Clearly $Z /(p) \subset A /(p)$. Using (4.1.8) (Fermat's Theorem), equality is established.

Let $F$ be the quotient field of $A$.

COROLlaRY 4.3. The orders on $A$ and on $F$ are the only orders under which they are totally ordered rings.

Proof. By (4.1.9) (Lagrange's Theorem), the order on $A$ is defined algebraically. Since squares must be non-negative in any totally ordered ring, the order on $A$ is unique. The order on $F$ uniquely determines, and is uniquely determined by, the order on $A$, proving the corollary. (Cf. [S].)

One of the most far-reaching results thus far obtained for $A$ is (4.1.2): i.e., that every finitely generated ideal in $A$ is principal. Such an integral domain will be called locally a principal ideal domain. $Z$ is such a domain that is not a principal ideal domain (5.7). Another such example is the algebra of all analytic functions on an open Riemann surface $\left[\mathrm{A}_{4}\right]$. One can readily check that (4.1.3), (4.1.5) and (4.1.6) hold for locally principal ideal domains.

Given an integral domain $B$ and a prime ideal $P$ in it, let $B_{P}=\{a / b: a \in B$ and $b \in B-P\} . B_{P}$ is, of course, a local ring whose maximal ideal $P_{P}$ is $\{a / b: a \in P$ and $b \in B-P\}$. Further, $a \rightarrow a / 1$ is an injective homomorphism of $B$ into $B_{P}$. (See $\left[\mathrm{ZS}_{1}\right.$, pp. 221-228] for details.) 
THEOREM 4.4. Let $B$ be locally a principal ideal domain and let $P$ be a prime ideal in $B . B_{P}$ is a valuation ring.

Proof. Let $K$ be the quotient field of $B$ and let $f \in K-B_{P}$. Then $f=a / b$, for $a, b \in B$ and $b \neq 0$. By hypothesis, $(a, b)=(d)$. Since $d \mid a$ and $d \mid b$, we may assume that $d=1$. Since $f \notin B_{P}, b \in P$. If $a \in P$, then $(a, b)=(1) \subset P$, a contradiction. Thus $a \notin P$ and $f^{-1} \in B_{P}$, proving that $B_{P}$ is a valuation ring.

Applying (4.4) to various classical results about localization $\left[\mathrm{ZS}_{1}\right.$, p. 228$]$ and the prime ideals in a valuation ring $\left[\mathrm{ZS}_{2}\right.$, p. 40$]$ allows us to conclude that the prime ideals of $B$ contained in $M$ form a chain, under inclusion, and are in natural, bijective, order-reversing correspondence with the convex subgroups of the value group of $B_{M}$. In $\S 6$ this group will be computed in case $B=Z(6.4)$.

As a consequence of the following theorem, we will see that $F$, the quotient field of $A$, is a nonstandard model of the rational number field $Q$.

THEOREM 4.5. Two objects in $\mathfrak{A}$ that are elementarily equivalent have elementarily equivalent quotient fields.

Frequently one reduces algebraic statements about say $Q$, to statements about $Z$. We will formalize this process into the following lemma, which has (4.5) as an immediate consequence.

LEMMA 4.6. Let $B$ be an object in $\mathfrak{A}$, let $K$ be its quotient field (again an object in $\mathfrak{U})$, let $a_{1}, \cdots, a_{n}, \cdots$ be in $B$, and let $b_{1}, \cdots, b_{n}, \cdots$ be positive elements in $B$. There exists a mapping $\phi \rightarrow \phi^{\prime}$ of $L(\mathfrak{A})$ into itself, such that $\phi\left(a_{1} / b_{1}, \cdots, a_{n} / b_{n}, \cdots\right)$ holds in $K$ if and only if $\phi^{\prime}\left(a_{1}, b_{1}, \cdots, a_{n}, b_{n}, \cdots\right)$ holds in $B$.

Proof. The atomic relation of equality in $K, E\left(a_{1} / b_{1}, a_{2} / b_{2}\right)$, meaning $a_{1} / b_{1}=a_{2} / b_{2}$, will be taken to $E^{\prime}\left(a_{1}, b_{1}, a_{2}, b_{2}\right)$, meaning $a_{1} b_{2}=a_{2} b_{1}$. Addition in $K$, expressed by $S\left(a_{1} / b_{1}, a_{2} / b_{2}, a_{3} / b_{3}\right)$, meaning $a_{1} / b_{1}+a_{2} / b_{2}=a_{3} / b_{3}$, will be taken to $S^{\prime}\left(a_{1}, b_{1}, a_{2}, b_{2}, a_{3}, b_{3}\right)$, meaning $a_{1} b_{2} b_{3}+a_{2} b_{1} b_{3}=a_{3} b_{1} b_{2}$. The product relation will be treated similarly. The order relation in $K, O\left(a_{1} / b_{1}, a_{2} / b_{2}\right)$, meaning $a_{1} / b_{1} \leqq a_{2} / b_{2}$, will be taken to $O^{\prime}\left(a_{1}, b_{1}, a_{2}, b_{2}\right)$, meaning $a_{1} b_{2} \leqq a_{2} b_{1}$. (Note: by hypothesis, $b_{1}$ and $b_{2}$ are positive.) Thus we have defined the mapping $\phi \rightarrow \phi^{\prime}$ on the atomic formulas. Let the mapping be extended by requiring that it preserve $\wedge$ and $\sim$. Assuming $\phi^{\prime}$ to be defined, let $\left(\left(\exists z_{n}\right) \phi\right)^{\prime}=\left(\exists x_{n}\right)\left(\exists y_{n}\right)\left(\phi^{\prime} \wedge\left(y_{n}>0\right)\right)$. One can easily verify that this mapping satisfies the conditions of the lemma.

Using (4.6), (4.5) follows immediately.

CoRollary 4.7. The quotient field $F$ of $A$ is a nonstandard model of $Q$. Thus $A$ is integrally closed in $F$.

Since the last statement is elementary and holds for $Z$, it holds for $A$.

The following is a special case of a theorem due to Frayne (see $[K, 9.1]$ ). 
THEOREM 4.8 (FRAYNE). Let $B$ and $B^{\prime}$ be objects in $\mathfrak{A}$ that are elementarily equivalent. There exists a discrete space $X, \mu \in \delta X$, and an order-preserving isomorphism that maps $B^{\prime}$ into $B \equiv$ ind $\lim _{U \in \mu} C(U, B)$. Finally, $X$ may be chosen to have the same cardinal number as $B^{\prime}$.

Using this, we can regard $A$ as a subdomain of $Z$ and $F$ as a subfield of $\boldsymbol{Q}=$ ind $\lim _{U \in \mu} C(U, Q)$.

Corollary 4.9. Any element in $F-Q$ is transcendental over $Q$.

Proof. Let $F$ be imbedded in $\boldsymbol{Q}$, let $\boldsymbol{f} \in F$, and assume that $\boldsymbol{f}$ is algebraic over $Q$. Let $m(t)=t^{n}+a_{1} t^{n-1}+\cdots+a_{n}$ be the minimal monic polynomial of $f$ in $Q[t]$. Let $f$ be a pre-image of $f$ in $C(X, Q)$. There exists $U \in \mu$ such that for all $x \in U, m(f(x))=0$. Let $q_{1}, \cdots, q_{k}$ be the distinct rational roots of $m(t)$ and let $U_{i}=\left\{x \in X: f(x)=q_{i}\right\}$. Clearly, $\left(U \cap U_{i}\right)_{i=1, \cdots, k}$ is a partition of $U$ by elements in $\mathscr{B}$. Since $U \in \mu$, one and only one $U \cap U_{i} \equiv V$ is in $\mu . f \mid V=q_{i}$, showing that $f \in Q$, proving the corollary.

REMARK. In spite of this corollary, $F$ is not a pure transcendental extension of $Q$, for the order on $F$ is unique (4.3).

5. On the structure of $Z$ and its quotient field. Since $Z$ is a nonstandard model of $Z$ (3.1), the results of $\S 4$ apply to $Z$. Since $Z$ has an explicit representation as ind $\lim _{U \in \mu} C(U, Z)$, one can translate questions on the existence of elements in $Z$ back to $C(X, Z)$, where elements can, in many cases, easily be constructed; thus when dealing with $Z$ we can go beyond the results of $\$ 4$.

An element $f$ in $C(X, Z)$ will be called a prime-valued function if $|f(x)| \in \Pi$ for all $x \in X$ (cf. [P, 4.3. 1]).

THEOREM 5.1. If $f$ is a prime-valued function in $C(X, Z)$, then $f$ is a prime in $Z$. Conversely, every prime $f$ in $Z$ has a prime-valued pre-image in $C(X, Z)$.

Proof. Let $f$ be a prime-valued function in $C(X, Z)$ and let $a, b \in C(X, Z)$ such that $f=a b$. There exists $U \in \mu$ such that $f|U=a b| U$. Let $V=a^{-1}( \pm 1) \cap U$ and $W=b^{-1}( \pm 1) \cap U$. Clearly, $V, W \in \mathscr{B}, U=V \cup W$, and $V \cap W=\varnothing$. Since $\mu$ is an ultrafilter in $\mathscr{B}$, either $V$ or $W$ is in $\mu$; accordingly, either $\boldsymbol{a}$ or $\boldsymbol{b}$ is a unit in $Z$, showing that $f$ is prime.

Conversely, assume that $f$ is a prime element in $Z$. We may assume, without loss of generality, that $f>0$. Let $g$ be a positive pre-image of $f$ in $C(X, Z)$. Since $f$ is not a unit, $X_{1} \equiv g^{-1}(1)$ is not in $\mu$. Since $X_{1} \in \mathscr{B}$ and $\mu$ is an ultrafilter in $\mathscr{B}$, $X-X_{1} \in \mu$. Assume for a moment that $X_{\Pi} \equiv g^{-1}(\Pi)$ is not in $\mu$. Let $N^{\prime}=N-(\Pi \cup\{1\})$. Clearly $g^{-1}\left(N^{\prime}\right) \equiv U$ is equal to $\left(X-X_{\Pi}\right) \cap\left(X-X_{1}\right)$ and is in $\mu$. For each $n \in N^{\prime}$, let $U_{n}=g^{-1}(n) .\left(U_{n}\right)_{n \in N^{\prime}}$ is a partition of $U$ by elements of $\mathscr{B}$. For each $n \in N^{\prime}$, there exist nonunits $a_{n}$ and $b_{n}$ in $N$ such that $n=a_{n} b_{n}$. Let $a\left|U_{n}=a_{n}, b\right| U_{n}=b_{n}$ for all $n \in N^{\prime}$, and $a|X-U=0=b| X-U$. 
Since $U \in \mu, \boldsymbol{f}=\boldsymbol{a} \boldsymbol{b}$. Further, by construction, $\boldsymbol{a}$ and $\boldsymbol{b}$ are nonunits in $\boldsymbol{Z}$. Since $\boldsymbol{f}$ is prime, this is a contradiction, and we conclude that $X_{\Pi} \in \mu$. Let $f\left|X_{\Pi}=g\right| X_{\Pi}$ and let $f \mid X-X_{\Pi}=2$. $f$ is a prime-valued function and $f \rightarrow f$, proving the theorem.

Before proceeding to consider corollaries of (5.1), we will need a technical result, which follows. Since $\mu$ is, by hypothesis, in $\delta X-\delta_{0} X, \mu$ does not have the countable intersection property. We will see (5.2) that this implies the existence of a family $\left(U_{n}\right)_{n \in N}$ of $\mu$ such that $U_{1}=X$, and $U_{n+1}<U_{n}$ for all $n \in N$. Let $V_{n}=U_{n}-U_{n+1}$ for all $n \in N$. Then $\left(V_{n}\right)_{n \in N}$ is a family of nonempty sets of $\mathscr{B}$ that partition $X$ and such that $\bigcup_{n \geqq m} V_{n} \in \mu$ for all $m \in N$. Such a partition of $X$ will be called a distinguished partition of $X$ relative to $\mu$.

LEMMA 5.2. Given $\mu \in \delta X$, there exists a distinguished partition of $X$ relative to $\mu$, if and only if $\mu \notin \delta_{0} X$.

Proof. Assume that $\mu \notin \delta_{0} X$. By the definition of $\delta_{0} X$, there exists $f \in Z-Z$. Assume that $\boldsymbol{f}>\mathbf{0}$ and let $f$ be a pre-image of $f$ in $C(X, Z)$ such that $f \geqq 1$. Let $U \equiv\{x \in X: n \leqq f(x)\}$. Clearly, $U_{n} \in \mathscr{B}, U_{1}=X, U_{n+1} \subset U_{n}$, and $U_{n} \in \mu$ Further, $\bigcap_{n \in N} U_{n}=\varnothing$, showing that, on reindexing $\left(U_{n}\right)_{n \in N}$, we can require that $U_{n+1}<U_{n}$. Let $V_{n}=U_{n}-U_{n+1}$; then $\left(V_{n}\right)_{n \in N}$ is a distinguished partition of $X$ relative to $\mu$. To prove the converse, let $\left(V_{n}\right)_{n \in N}$ be such a partition, and let $f \mid V_{n}=n$ for all $n$. Then $f \in Z-Z$, showing that $\mu \notin \delta_{0} X$, proving the lemma.

Let $c$ denote the power of the continuum.

COROLlary 5.3. There exist at least $\mathrm{c}$ infinite primes in $\mathrm{Z}$.

Proof. Let $\left(V_{n}\right)_{n \in N}$ be a distinguished partition of $X$ relative to $\mu$. Let $\Pi$ be partitioned into two infinite sets, $\Pi_{0}$ and $\Pi_{1}$, and let $p_{i}$ be the least element of $\Pi_{i}$. Let $f_{i} \mid V_{1}=p_{i}$. Let $\Pi_{i}-\left\{p_{i}\right\}$ be partitioned into two infinite subsets, $\Pi_{i, j}$, and let $p_{i, j}$ be the least element of this set. Let $f_{i, j}$ extend $f_{i}$ and let $f_{i, j} \mid V_{2}=p_{i, j}$. Continuing in this fashion, by finite induction, one defines prime-valued functions $f_{\tau}$ in $C(X, Z)$ for each $\tau \in\{0,1\}^{N}$. By (5.1), $f_{\tau}$ is a prime in $Z$. Since $\left(V_{n}\right)_{n \in N}$ is distinguished, $U_{m} \equiv \bigcup_{n \geqq m} V_{n}$ is in $\mu$. Since $f_{\tau} \mid U_{m} \geqq m, f_{\tau}$ is not in $Z$. Given distinct $\tau$ and $\sigma \in\{0,1\}^{N}$, let $m$ be the least integer for which $\tau(m) \neq \sigma(m)$. By construction, $f_{\tau}\left(U_{m}\right) \cap f_{\sigma}\left(U_{m}\right)=\varnothing$, showing that $f_{\tau} \neq f_{\sigma}$, proving the corollary.

REMARK. Let $\phi_{n}=(\exists x)(x \neq n)$, for $n \in Z$. To the sentences in $L(\mathfrak{A})$ used to define $Z$, adjoin $\left(\phi_{n}\right)_{n \in Z}$. The resulting axiom system is for nonstandard models of $Z$. By the Löwenheim-Skolem theorem [R, 1.5.13], there is a countable nonstandard model $A$ of $Z$. By (5.3), $A$ is not isomorphic to $Z$.

Let $f$ be a prime-valued function in $C(X, Z)$ and let $M(f, \mu)=\{g \in C(X, Z)$ : $D(f) \cap(U \times \Pi) \subset D(g)$, for some $U \in \mu\}$. It has been shown that $M(f, \mu)$ is a maximal ideal in $C(X, Z)[\mathrm{P}, 4.3 .4]$. By (5.1) and (4.1.6), we arrive at another corollary. 
COROLlary 5.4. Let $f$ be a prime-valued function in $C(X, Z)$. Then $M(f, ! \mu)$ is the pre-image of the maximal ideal $(f)$ in $Z$.

Let $\mathfrak{m}_{f}$ be the set of finite maximal ideals of $\boldsymbol{Z}$ (i.e., $\{\boldsymbol{M} \notin \mathfrak{m}: M \cap Z$ is a maximal ideal in $Z\}$ ), and let $m_{p}$ be the set of all principal maximal ideals of $Z$. Clearly, $m_{f} \subset m_{p} \subset m$. By (5.3), we know that $m_{p}-m_{f}$ is of power at least $c$, and by (4.1.7) we know that $m_{p}$ is dense in $m$.

Corollary 5.5. $\mathrm{m}_{f}$ is closed in $\mathrm{m}_{p}$.

Proof. Recall that the closure of $\mathrm{m}_{f}$ in $\mathrm{m}$ is $\left\{M \in \mathfrak{m}: \bigcap_{p \in \Pi(p) \subset M}\right\}$. To prove this corollary, it suffices to show that, given any infinite prime $f$ in $Z$, there exists $g \in \cap_{p \in \Pi}(p)$ such that $g \notin(f)$. Without loss of generality, we may assume that $f>0$. Let $f$ be a positive, prime-valued pre-image of $f(5.1)$. Let $X_{p}=f^{-1}(p)$ and note that $\left(X_{p}\right)_{p \in \Pi}$ is a partition of $X$ by elements of $\mathscr{B}$. Since $f \notin Z$, no $X_{p}$ is in $\mu$, Let $(p(n))_{n \in N}$ be a proper indexing of $\Pi$. Let $g \mid X_{p(1)}=1$ and, for $\mathrm{n}>1$, let $g \mid X_{p(n)}=p(1) \cdot \cdots \cdot p(n-1)$. Then $g \in C(X, Z),(g, f)=(1)$ and thus $(g, f)=(1)$, showing that $g \notin(f)$. Since $\mu$ is an ultrafilter in $\mathscr{B}$ and since $X_{p} \notin \mu$ for any $p \in \Pi$, given $m \in N$, then $\bigcup_{1 \leqq n \leqq m} X_{p(n)}$ is not in $\mu$. Since $\mu$ is an ultrafilter in $\mathscr{B}$, there exists $U \in \mu$ such that $\left(\bigcup_{1 \leqq n \leqq m} X_{p(n)}\right) \cap U=\varnothing$. Given $x \in U, x \in X_{p(j)}$ implies $j>m$; thus $p(1), \cdots, p(m)$ divide $g(x)$, showing that $g \in(\boldsymbol{p})$ for all $p \in \Pi$, proving the corollary.

The mapping $p \rightarrow(p)$ is a bijection between $\Pi$ and $m_{f}$. As the maximal ideal space of $Z, \Pi$ is a compact $T_{1}$-space, a base for the closed sets of $\Pi$ being its finite subsets, The next result implies that, under this topology, $p \rightarrow(p)$ is not continuous.

Proposition 5.6. The topology of $\mathrm{m}_{f}$, induced from $\mathrm{m}$, is the discrete topology.

Proof. Since $Z \subset Z$, all finite subsets of $m_{f}$ are closed in $m_{f}$. Let $m_{f}^{\prime}$ be an infinite subset of $m_{f}$ and let $\Pi^{\prime}$ be the pre-image of $m_{f}^{\prime}$ in $\Pi$. Let $(p(n))_{n \in N}$ be a proper indexing of $\Pi^{\prime}$ and let $\left(V_{n}\right)_{n \in N}$ be a distinguished partition of $X$ relative to $\mu$. Let $f \mid V_{n}=p(1) \cdot \cdots \cdot p(n)$. Then $f \in C(X, Z), f \in Z-Z$, and $\left\{\boldsymbol{M} \in \mathrm{m}_{\boldsymbol{f}}: \boldsymbol{f} \in \boldsymbol{M}\right\}=\mathrm{m}_{\boldsymbol{f}}^{\prime}$, proving the proposition.

CoROllary 5.7. $m_{p} \neq m$.

Proof. Were $\mathfrak{m}_{p}=\mathfrak{m}$, then $\mathfrak{m}_{p}$ would be compact. Since $\mathfrak{m}_{f}$ is closed in $\mathfrak{m}_{p}$ (5.5), $\mathfrak{m}_{f}$ would be compact. Since $\mathfrak{m}_{f}$ has the discrete topology (5.6), it would have to be finite, a contradiction.

Since $\mathfrak{m}_{f}$ is closed in $\mathfrak{m}_{p}(5.5),\left(\mathrm{cl}_{\mathfrak{m}} \mathrm{m}_{f}\right)-\mathfrak{m}_{f} \subset \mathfrak{m}-\mathfrak{m}_{p}$. Since $\mathfrak{m}_{f}$ is a discrete space (5.6) and $m$ is compact, the boundary of $m_{f}$ in $m$ is nonempty.

THEOREM 5.8. $\left(\mathrm{cl}_{\mathfrak{m}} \mathrm{m}_{f}\right)-\mathrm{m}_{f}$ is a proper subset of $\mathfrak{m}-\mathfrak{m}_{p}$. 
Proof. Let $\left(V_{n}\right)_{n \in N}$ be a distinguished partition of $X$ relative to $\mu$. Let $(p(n))_{n \in N}$ be an enumeration of $\Pi$, and let $f \mid V_{n}=p(1) \cdot \cdots \cdot p(n)$. Then $\boldsymbol{f} \in \bigcap_{\boldsymbol{p} \in \boldsymbol{\Pi}}(\boldsymbol{p})$. We will use [P, 4.4] to construct $\boldsymbol{M} \in \mathbb{m}-\mathfrak{m}_{p}$ such that $\boldsymbol{f} \notin \boldsymbol{M}$. Let $\Phi_{n}=\{p(m): n<m<2 n\}$ and let $B=\bigcup_{n \in N} V_{n} \times \Phi_{n}$. We will show that $\mathfrak{b}=\left\{B \cap\left(\boldsymbol{D}\left(f_{1}\right)\right)^{\mathfrak{c}} \cap \cdots \cap\left(\boldsymbol{D}\left(f_{k}\right)\right)^{\mathfrak{c}} \cap U \times \Pi: f_{1}, \cdots, f_{k}\right.$ being prime-valued functions in $C(X, Z)$ and $U \in \mu\}$ is a filter base in $\mathscr{D} \equiv D(C(X, Z))$, where the superscript c means complementation. Since $\left(V_{n}\right)_{n \in N}$ is a distinguished partition of $X$ relative to $\mu$, given $m>k, U_{m} \equiv \bigcup_{n \geqq m} V_{n}$ is in $\mu$. Given $x \in U_{m}, x \in V_{n}$ for some $n>k$. Among the $n$ primes of $\Phi_{n}$ there must be one which is distinct from $f_{1}(x), \cdots, f_{k}(x)$, showing that $\mathfrak{b}$ is a filter base of $\mathscr{D}$. Let $D$ be an ultrafilter in $\mathscr{D}$ that contains $\mathfrak{b}$, and let $M=D^{-1}(\mathfrak{b})$. By construction, $\sigma(M)=\mu$. Since $\boldsymbol{D}(f) \cap B=\varnothing$, $f \notin M$. By construction, $M \notin \mathfrak{m}_{p}$, proving the theorem.

Remark. Pierce $[\mathrm{P}, 5.2]$ has shown that any fixed cardinal number can be exceeded by the power of $C(X, Z) / M$, for suitable $X$ and $M$. A fortiori, a $Z$ can be found whose power exceeds any given cardinal number. The following is a variant of this argument.

Proposition 5.9. Let $X$ be a discrete space of infinite power $\mathfrak{n}$. There exists $\mu \in \delta X-\delta_{0} X$ such that $\mathfrak{m}_{p}$ has power greater than $\mathfrak{n}$.

Proof. Let $\left(F_{x}\right)_{x \in X}$ be a proper indexing of the nonempty finite subsets of $X$. Given $y \in X$, let $X_{y}=\left\{x \in X: y \in F_{x}\right\}$. The family $\left(X_{y}\right)_{y \in X}$ has the finite, but not the countable intersection property. Let $\mu$ be an ultrafilter on $X$ containing $\left(X_{y}\right)_{y \in X}$; then $\mu \notin \delta_{0} X$. Let $Z=C(X, Z) / P_{\mu}$. Assume for a moment that the power of $\mathfrak{m}_{p}$ does not exceed $\mathfrak{n}$. Let $\mathfrak{m}_{p}=\left(\left(g_{y}\right)\right)_{y \in X}$. By (5.1), choose a prime-valued pre-image $g_{y}$ in $C(X, Z)$ of each prime $g_{y}$. Since $F_{x}$ is a nonempty finite set, $\left\{g_{y}(x)\right\}_{y \in F_{x}}$ is a finite set of prime numbers. Let $f(x)$ be the least prime number greater than all $g_{y}(x), y \in F_{x}$. By construction, $f$ is a prime-valued function and, by (5.1), $\boldsymbol{f}$ is a prime in $\boldsymbol{Z}$. Since $g_{y}\left|X_{y}<f\right| X_{y}$ and since $X_{y} \in \mu, g_{y}<f$, showing that $(f) \notin \mathfrak{m}_{p}$, a contradiction, proving that the power of $\mathfrak{m}_{p}$ exceeds $\mathfrak{n}$. (Cf. [GJ, 12.7].)

Given $f \in C(X, Q), Q$ having the discrete topology, there exist unique $a$, $b \in C(X, Z)$ such that $b>0,(a, b)=(1)$, and $f=a / b$. Clearly, $f=a / b,(a, b)=(\mathbf{1})$, and $\boldsymbol{b}>\mathbf{0}$. Whenever we write $f(f)$ as a quotient of elements of $C(X, Z)(Z)$, it will be assumed, unless otherwise stated, that such a quotient has this form. Clearly, $Q \equiv$ ind $\lim _{U \in \mu} C(U, Q)$ is the quotient field of $Z$ in $Q$.

We will close the section with an analysis of the order structure of $\boldsymbol{Q}$ and $\boldsymbol{Z}$. Let $T$ be a nonempty, totally ordered set and let $A$ and $B$ be subsets of $T$. One writes $A<B(A \leqq B)$ if given $a \in A$ and $b \in B$, then $a<b(a \leqq b) . T$ is called an $\eta_{1}$-set $\left[\mathrm{H}_{1}\right.$, p. 180] (a near $\eta_{1}$-set $\left[\mathrm{A}_{3}, \S 4\right]$ ) if given two countable (nonempty) subsets $A$ and $B$ of $T$ such that $: A<B(A \leqq B)$, there exists $t \in T$ such that $A<t<B(A \leqq t \leqq B)$. 
THEOREM 5.10. $Z$ is a near $\eta_{1}$-set in which no countable set is cofinal. $Q$ is an $\eta_{1}$-set.

Proof. Let $\boldsymbol{A}$ and $\boldsymbol{B}$ be two nonempty, countable subsets of $\boldsymbol{Z}(Q)$ such that $\boldsymbol{A}<\boldsymbol{B}$. Using [GJ, 13.5], we can choose $\left(f_{n}\right)_{n \in N}$ and $\left(g_{n}\right)_{n \in N}$ in $C(X, Z)(C(X, Q))$ such that $f_{k} \leqq f_{n} \leqq g_{n} \leqq g_{k}$ whenever $k<n,\left(f_{n}\right)_{n \in N}$ is cofinal in $A$, and $\left(g_{n}\right)_{n \in N}$ is coinitial in $B$. Let $\left(V_{n}\right)_{n \in N}$ be a distinguished partition of $X$ relative to $\mu$ and let $t\left|V_{n}=f_{n}\right| V_{n}$ for all $n \in N$. Clearly, $t$ is in $C(X, Z)(C(X, Q))$. Further, $\boldsymbol{f}_{k} \leqq \boldsymbol{t} \leqq \boldsymbol{g}_{\boldsymbol{k}}$ for all $k \in N$, showing that $\boldsymbol{Z}$ and $\boldsymbol{Q}$ are near $\eta_{1}$-sets. To show that $\boldsymbol{Z}$ has no countable cofinal set, omit $\boldsymbol{B}$ from the construction above. Applying [GJ, 13.8] shows that $Q$ is, in addition, an $\eta_{1}$-set, proving the theorem.

REMARK. Since $\boldsymbol{Q}$ is an $\eta_{1}$-set, the residue class field of its order valuation is the reals $\left[\mathrm{A}_{1}\right],\left[\mathrm{A}_{2}\right]$. However, by (4.9), $Q$ contains no subfield of coset representatives of this field.

6. Valuations of $Q$ associated with maximal ideals of $Z$. We have seen (4.4) that, given a maximal ideal $M$ of $Z, Z_{M}$ is a valuation ring of $Q$. It will be shown (6.3) that the value group of this valuation can be computed in the following roundabout fashion: by means of a divisor map $d$, we can go from $C(X, Q)^{*}$ to $Z^{X \times \Pi}$, and then pass, by means of an inductive limit along $D(M)$, to a totally ordered group $G$. We will see that $Q^{*}$ maps onto $G$ in such a way as to give the desired valuation. (Note the similarity between this and the method of obtaining value groups in $\left[\mathrm{A}_{4}, \S 2\right]$.) Having done this, it will be quite easy to study $G$.

Since $\boldsymbol{Z}_{\boldsymbol{M}}$ is a local ring of $\boldsymbol{Q}$ whose maximal ideal is $\boldsymbol{M}_{\boldsymbol{M}}$, the group of units $\boldsymbol{U}$ of $\left(\boldsymbol{Z}_{M}\right)^{*}$ is $\boldsymbol{Z}_{\boldsymbol{M}}-\boldsymbol{M}_{\boldsymbol{M}}$. Recall that a valuation of $\boldsymbol{Q}$ associated with $\boldsymbol{Z}_{\boldsymbol{M}}$ is a homomorphism $V$ of the multiplicative group of $Q^{*}$, whose kernel is $U$. Let $\Gamma$ be the range of $V$. Since $\boldsymbol{Z}_{M}$ is a valuation ring, $V\left(Z^{*}\right)$ serves as a set of non-negative elements $(\equiv \Gamma(\geqq 0))$ of $\Gamma$, and makes $\Gamma$ into a totally ordered group. Extend $V$ to $Q$ by letting $V(\mathbf{0})=\infty, \infty$ being a symbol greater than all elements in $\Gamma$, such that $\infty+\gamma=\gamma+\infty=\infty$ for all $\gamma \in \Gamma$. Then $V(\boldsymbol{a} \pm \boldsymbol{b}) \geqq \min \{V(\boldsymbol{a}), V(\boldsymbol{b})\}$, and $V(a b)=V(a)+V(b)$ for all $a$ and $\boldsymbol{b}$ in $Q$.

Given $f \in C(X, Q)$, let $d(f)$ be the following mapping defined on $X \times \Pi$ : given $(x, p) \in X \times \Pi$, let $d(f)(x, p)=v_{p}(f(x))$, where $v_{p}$ is the $p$-adic valuation on $Q$. Clearly, $d$ is a homomorphism of $C(X, Q)^{*}(\equiv\{f \notin C(X, Q): f(x) \neq 0$ for all $x \in X\})$ into $Z^{X \times \Pi}$. Let the range of $d$ be given pointwise addition and order. Then $d(f \pm g) \geqq d(f) \wedge d(g)$, and $d(f g)=d(f)+d(g)$. Clearly, $f \in C(X, Z)$ if and only if $d(f) \geqq 0$.

By the support of $d(f)$ is meant the set $\{(x, p) \in X \times \Pi: d(f)(x, p) \neq 0\}$.

Proposition 6.1. Let $a \in C(X, Z) . D(a)$ is the support of $d(a)$.

Proof. It suffices to observe that the following are equivalent: $(x, p) \in \boldsymbol{D}(a)$, $a(x) \equiv 0(p), v_{p}(a(x))>0$, and $d(a)(x, p)>0$. 
Let $\lambda$ be the canonical homomorphism of $d C(X, Q)^{*}$ onto

$$
\text { ind } \lim _{m \in M} d C(X, Q)^{*} \mid D(m) \equiv G .
$$

Since $d C(X, Q)^{*} \mid \boldsymbol{D}(m)$ is a partially ordered group, $G$ is a partially ordered group and $\lambda$ is an order-preserving mapping.

LEMMA 6.2. $d C(X, Q)^{*}$ is a lattice-ordered subgroup of $Z^{X \times \Pi}, G$ is a totally ordered group, $\lambda d C(X, Z)^{*}=G(\geqq 0)$, and the kernel $U$ of $\lambda d$ is $\left\{a \mid b: a, b \in C(X, Z)^{*}-M\right\}$.

Proof. Let $f \in C(X, Q) *$ equal $a / b$, where

$$
a, b \in C(X, Z)^{*}(=\{g \in C(X, Z): \zeta(g)=\varnothing\}) .
$$

By convention, $(a, b)=(1)$; thus $d(f)=d(a)-d(b)$, where $d(a), d(b) \geqq 0$ and $d(a) \wedge d(b)=0$. Using [B, p. 215], one sees that $d C(X, Q) *$ is a lattice-ordered group. Since $(a, b)=(1)$, at most one of $a$ and $b$ is in $M$ : i.e., at most one of $D(a)$ and $\boldsymbol{D}(b)$ is in $\boldsymbol{D}(M)$. By $(6.1), \boldsymbol{D}(a)(\boldsymbol{D}(b))$ is the support of $d(a)(d(b))$. Thus $a \in M$ if and only if $\lambda d(f)=\lambda d(a)>0, b \in M$ if and only if $\lambda d(f)=-\lambda d(b)<0$, and $a$ and $b$ are not in $M$ if and only if $\lambda d(f)=0$, proving the lemma.

Let $\eta$ be the canonical homomorphism of $C(X, Q)^{*}$ onto $Q^{*}$.

THEOREM 6.3. There exists a unique, order-preserving isomorphism $\theta$ of $\Gamma$ onto $G$ such that the following diagram is commutative:

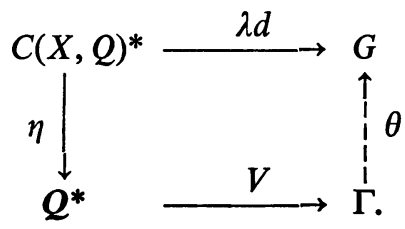

Proof. By (6.2), the kernel $U$ of $\lambda d$ is $\left\{a / b: a, b \in C(X, Z)^{*}-M\right\}$. By definition, the kernel $U$ of $V$ is $Z_{M}-M_{M}$ : i.e., $\left\{a / b: a, b \in Z^{*}-M\right\}$, showing that $\eta^{-1}(U)=U$. We conclude that a unique group isomorphism $\theta$ of $\Gamma$ into $G$ exists, making the diagram commutative. Since $\lambda d$ is surjective, $\theta$ is surjective. By (6.2), $\lambda d C(X, Z)^{*}=G(\geqq 0), \eta C(X, Z)^{*}=Z^{*}$, and $V\left(Z^{*}\right)=\Gamma(\geqq 0)$, showing that $\theta$ is, order preserving, proving the theorem.

Let $V=\theta V$ and let $V(0)=\infty$. Then $V$ is a valuation of $Q$ whose valuation ring is $Z_{M}$ and whose value group is $G$. Let us now investigate the structure of $G$.

THEOREM 6.4. $G$ is a near $\eta_{1}$-set, with a least positive element $g_{0}$, in which no countable set is cofinal. If $M=(f)$, then $V(f)=g_{0}$.

Proof. Pierce has observed [P, Proof of 4.5.4] that there exists $m \in M$ such that for all $x \in X$, either $m(x)=1$ or $m(x)$ is the product of distinct primes. Thus $d(m) \mid D(m)=1$, and $\lambda d(m)$ is $g_{0}$. Note that if $M$ contains a prime-valued function 
$f$ (i.e., if $\boldsymbol{M}=(\boldsymbol{f})$ ), then $\lambda d(f)$ is $g_{0}$, showing that $V(f)=g_{0}$. Let $A$ and $B$ be nonempty, countable subsets of $G$ such that $A \leqq B$. Since $d C(X, Q)^{*}$ is a latticeordered group, we may invoke [GJ, 13.5] and obtain $f_{n}$ and $g_{n} \in C(X, Q)^{*}$ such that the following conditions hold: $d\left(f_{k}\right) \leqq d\left(f_{n}\right) \leqq d\left(g_{n}\right) \leqq d\left(g_{k}\right)$ for all $k<n$, $\left(\lambda d\left(f_{n}\right)\right)_{n \in N}$ is cofinal in $A$, and $\left(\lambda d\left(g_{n}\right)\right)_{n \in N}$ is coinitial in $B$. Let $\left(V_{n}\right)_{n \in N}$ be a distinguished partition of $X$ relative to $\mu$ (5.2). Let $t\left|V_{n}=f_{n}\right| V_{n}$ for all $n \in N$; then $t \in C(X, Q)^{*}$. By definition, $\bigcup_{n \geqq k} V_{n} \equiv U_{k}$ is in $\mu$. Since $P\left(\equiv P_{\mu}\right)$ is the minimal prime ideal contained in $M$, there exists $m \in M$ such that $U_{k}=\mathscr{U}(m)(=\{x \in X$ : there exists $p \in \Pi$ such that $(x, p) \in D(m)\})$. Let $(x, p) \in D(m)$; then $x \in V_{n}$ for some $n \in N$. Since $\mathscr{U}(m)=U_{k}, n \geqq k$. Clearly,

$$
d\left(f_{k}\right)(x, p) \leqq d\left(f_{n}\right)(x, p)=d(t)(x, p) \leqq d\left(g_{n}\right)(x, p) \leqq d\left(g_{k}\right)(x, p),
$$

showing that $d\left(f_{k}\right)|\boldsymbol{D}(m) \leqq d(t)| \boldsymbol{D}(m) \leqq d\left(g_{k}\right) \mid \boldsymbol{D}(m)$. Thus $\lambda d\left(f_{k}\right) \leqq \lambda d(t) \leqq \lambda d\left(g_{k}\right)$ for all $k \in N$, showing that $A \leqq \lambda d(t) \leqq B$, proving that $G$ is a near $\eta_{1}$-set. To prove the last assertion of the theorem, let $B=\varnothing$ and proceed as above, proving the theorem. (Cf. $\left[\mathrm{A}_{4}, 2.6\right]$.)

A subset $G^{\prime}$ of $G$ will be called convex if, given $g^{\prime} \in G^{\prime}$ and $g \in G$ such that $|g| \leqq\left|g^{\prime}\right|$, then $g \in G^{\prime}$. The convex subgroups of $G$ form a totally ordered set under inclusion. Given $g \in G$ let $W(g)$ be the smallest convex subgroup of $G$ that contains $g$. Clearly, $W(g \pm h) \leqq W(g) \cup W(h)$ for all $g, h \in G$. W is called the order valuation on $G$. Let $S=\left\{W(g): g \in G^{*}\right\}$. S, a totally ordered set, is called the value set of $G$. The convex subgroups of $G$ are in bijective, order-preserving correspondence with the lower sets of $S$ under the mapping $G^{\prime} \rightarrow W\left(G^{\prime}\right) \cap S$. (See, e.g., $\left[\mathrm{A}_{2}\right]$ for references.)

Applying $\left[\mathrm{A}_{3}, 5.1\right.$ (ii) $]$ and (6.4) we arrive at the following.

Corollary 6.5. The value set $S$ of $G$ has a least element $s_{0}$, and $S-\left\{s_{0}\right\}$ is an $\eta_{1}$-set. Thus the nonzero convex subgroups of $G$ are in natural bijective correspondence with the Dedekind completion $S$ of $S$.

Given $s \in S$, let $W^{-1}(\leqq s)\left(W^{-1}(<s)\right)=\{g \in G: W(g) \leqq s\} \quad(=\{g \in G: W(g)<s\})$. Both $W^{-1}(\leqq s)$ and $W^{-1}(<s)$ are convex subgroups of $G$. Further, $W^{-1}(<s)$ is the largest proper, convex subgroup of $W^{-1}(\leqq s)$. Let $H(s) \equiv W^{-1}(\leqq s) / W^{-1}(<s)$, inherit the order on $W^{-1}(\leqq s)$. $H(s)$ is then an Archimedian totally ordered group, and hence can be imbedded in the additive group of real numbers. (See, e.g., $\left[A_{2}\right]$ for references.)

THEOREM 6.6. $H(s)$ is isomorphic to the integers if $s=s_{0}$, and to the reals if $s>s_{0}$.

Proof. If $s=s_{0}$, then $H(s)$ is isomorphic to the subgroup of $G$ generated by $g_{0}$, the least positive element of $G$, proving the first assertion. Assume that $s>s_{0}$. It will first be shown that every element in $H(s)$ is divisible by 2 in $H(s)$. Let $h \in H(s)$ 
be positive, and let $g$ be a pre-image of $h$ in $W^{-1}(\leqq s)$. Since $g \geqq 0$, there exists $f \in C(X, Z)^{*}$ such that $f>0$ and $\lambda d(f)=g$. Since $g>0, f \in M$ (6.1); thus $U \equiv \mathscr{U}(f) \in \mu$. Let $V$ equal $\left\{x \in U: v_{p}(f(x)) \equiv 0(2)\right.$ for all $\left.p \in \Pi\right\}$. To see that $V \in \mathscr{B}$, note that $V=U \cap f^{-1}\left\{n \in N: v_{p}(n) \equiv 0(2)\right.$ for all $\left.p \in \Pi\right\}$. If $V \in \mu$, let $a\left|V=f^{1 / 2}\right| V$ and let $a \mid X-V=1$. By the definition of $V, a \in C(X, Z)^{*}$. $2 \lambda d(a)=\lambda d(f)=g$, showing that $h$, the image of $g$ in $H(s)$, is divisible by 2 in $H(s)$. Assume now that $V \notin \mu$. Since $U \in \mu$, and $\mu$ is an ultrafilter in $\mathscr{B}, Y \equiv U-V$ is in $\mu$. Let $Y_{n}=Y \cap f^{-1}(n)$ for all $n \in N$. $\left(Y_{n}\right)_{n \in N}$ is a partition of $Y$ by elements of $\mathscr{B}$. Given $x \in Y$, there exist $n \in N$ such that $x \in Y_{n}$. Let $n=p_{1}^{e_{1}} \cdots \cdots p_{k}^{e_{k}}$, where $e_{1}, \cdots, e_{k} \geqq 1, e_{1}, \cdots, e_{r} \equiv 0(2)$, and $e_{r+1}, \cdots, e_{k} \equiv 1(2)$. Let

$$
a \mid Y_{n}=p_{1}^{e_{1} / 2} \cdots \cdots p_{r}^{e_{r} / 2} p_{r+1}^{\left(e_{r+1}-1\right) / 2} \cdots \cdots \cdot p_{k}^{\left(e_{k}-1\right) / 2},
$$

and let $b \mid Y_{n}=p_{r+1} \cdots \cdots p_{k}$, for all $n \in N$. Let $a|X-Y=b| X-Y=1$. Then $a, b \in C(X, Z)^{*} . g=\lambda d(f)=\lambda d\left(a^{2} b\right)=2 \lambda d(a)+\lambda d(b)$. Since $0 \leqq d(b) \leqq 1, \lambda d(b)$ is either zero or the least positive element of $G$. In either case, $\lambda d(b) \in W^{-1}(<s)$, since $s>s_{0}$. Thus $h$ is divisible by 2 in $H(s)$. Since every element of $H(s)$ is divisible by two in $H(s), H(s)$ is not isomorphic to the integers. By (6.4) and $\left[\mathrm{A}_{3}, 5.1\right.$ (i)], $H(s)$ is order isomorphic to the additive group of real numbers, proving the theorem.

Having studied $G$ in complete generality, let us now assume that $\boldsymbol{M}$ is a principal ideal $(f)$. Let $f$ be a prime-valued pre-image of $f$ in $C(X, Z)$. By $(6.4), \lambda d(f)$ is the least positive element of $G$. We will define $\boldsymbol{f}^{\boldsymbol{h}}$ for all $\boldsymbol{h} \in \boldsymbol{Z}$ and show that $\boldsymbol{h} \rightarrow \boldsymbol{V}\left(\boldsymbol{f}^{\boldsymbol{h}}\right)$ is an order-preserving isomorphism of the additive group of $\boldsymbol{Z}$ onto $G$ (6.9). Elements of the form $\boldsymbol{f}^{\boldsymbol{h}}$, for $\boldsymbol{h}>\mathbf{0}$, will also be of use in studying the ideal theory of $\boldsymbol{Z}$ in $\$ 7$.

Let $f \in C(X, Q)^{*}$, let $h \in C(X, Z)$, let $U_{q}=f^{-1}(q)$ for all $q \in Q^{*}$, and let $V_{n}=h^{-1}(n)$ for all $n \in Z$. Since $U_{q} \cap V_{n}$ is in $\mathscr{B}$, letting $f^{h} \mid U_{q} \cap V_{n}=q^{n}$ defines $f^{h}$, an element in $C(X, Q)^{*}$. Given $g \in C(X, Q)^{*}$ and $k \in C(X, Z)$, then $f^{h+k}=f^{h} f^{k}$ and $(f g)^{h}=f^{h} g^{h}$. Let $f^{h}$ be the image of $f^{h}$ in $Q^{*}$. Note that $f^{h}$ is dependent only on $f \in Q^{*}$ and $h \in Z$. Clearly, $f^{h+k}=f^{h} f^{k}$ and $(f g)^{h}=f^{h} g^{h}$, showing that $Q^{*}$ is a unitary $Z$-module.

We digress a bit to give the following.

Proposition 6.7. Let $\boldsymbol{f}$ be a prime element of $\boldsymbol{Z}$, and let $\boldsymbol{h}$ be a positive element in $\boldsymbol{Z}$. The only maximal ideal of $\boldsymbol{Z}$ that contains $\boldsymbol{f}^{\boldsymbol{h}}$ is $(\boldsymbol{f})$.

Proof. Since $\boldsymbol{f}^{\boldsymbol{h}}=\boldsymbol{f}^{\boldsymbol{h}-1} \boldsymbol{f}$, and $\boldsymbol{h}-\mathbf{1} \geqq \mathbf{0}, \boldsymbol{f}^{\boldsymbol{h}} \in(\boldsymbol{f})$. Let $f$ be a prime-valued preimage of $f$ in $C(X, Z)(5.1)$, and let $h$ be a positive pre-image of $\boldsymbol{h}$ in $C(X, Z)$. Then, by definition, $f^{h} \rightarrow f^{h}$. Since $D\left(f^{h}\right)=D(f), f^{h}$ in a maximal ideal $M$ implies $f \in M$, proving the proposition.

Let us now make $Z^{X \times \Pi}$ into a unitary $C(X, Z)$-module as follows: given $h \in C(X, Z)$ and $\alpha \in Z^{X \times \Pi}$, let $(h \alpha)(x, p)=h(x) \alpha(x, p)$. If $\alpha \geqq 0$, then $h \rightarrow h \alpha$ 
is order preserving. Since $h(x)(d(f)(x, p))=h(x) v_{p}(f(x))=v_{p}\left(f^{h}(x)\right)=d\left(f^{h}\right)(x, p)$, we see that $d C(X, Q)^{*}$ is a submodule of $Z^{X \times \Pi}$. Further, the $C(X, Z)$-module structure on $d C(X, Q)^{*}$ induces a natural unitary $Z$-module structure on $G$ such that, given $g \geqq 0, \boldsymbol{h} \rightarrow \boldsymbol{h} g$ is order preserving. Clearly, $\lambda(h d(f))=\boldsymbol{h} \lambda d(f)$, which, in turn, is $\lambda d\left(f^{h}\right)$. Utilizing the $Z$-module structure on $Q^{*}$ and (6.3), we see that $\boldsymbol{V}\left(\boldsymbol{f}^{\boldsymbol{h}}\right)=\boldsymbol{h} \boldsymbol{V}(\boldsymbol{f})$ : i.e, $\boldsymbol{V}$ is a $Z$-homomorphism. We will now summarize these results.

Proposition 6.8. $G$ is a unitary $Z$-module. Given $g \geqq 0$, the mapping $\boldsymbol{h} \rightarrow \boldsymbol{h} \boldsymbol{g}$ is an order-preserving mapping of $Z$ into $G$. $V$ is a $Z$-homomorphism of $Q^{*}$ onto $G$.

Unfortunately, (6.8) does not shed much additional light on the relation between $\boldsymbol{Z}$ and $\boldsymbol{G}$. However, if $\boldsymbol{M}$ is principal, more can be deduced.

THEOREM 6.9. Let $M$ be the principal ideal $(f)$. Then $V(f)$ is a generator of $G$ as a $\mathbf{Z}$-module and $\boldsymbol{h} \rightarrow \boldsymbol{h} \boldsymbol{V}(\boldsymbol{f})$ is an order-preserving isomorphism of the additive group of $\boldsymbol{Z}$ onto $G$.

Proof. Let $f$ be a prime-valued pre-image of $f$ in $C(X, Z)$ (5.1). By (5.4), $M=M(f, \mu)$; thus $\{D(f) \cap(U \times \Pi): U \in \mu\}$ is a base for $\boldsymbol{D}(M)$. Let $g$ be a positive element of $G$ and let $a \in C(X, Z)^{*}$ such that $\lambda d(a)=g$ (6.2). Since $g>0$, there exists $U \in \mu$ such that $d(a) \mid D(f) \cap(U \times \Pi)>0$. Given $x \in U$, then, $f(x)$ is prime and $v_{f(x)}(a(x))>0$, showing that $a(x)$ is a multiple of $f(x)$. Let $U_{n}=a^{-1}(n)$ for all $n \in Z^{*}$ and let $V_{p}=f^{-1}(p)$ for all $p \in \Pi$. Let $h \mid U_{n} \cap V_{p} \cap U=v_{p}(n)$ and let $h \mid X-U=0$. Given $x \in U, x$ is in $U_{a(x)} \cap V_{f(x)}$. Then

$$
d(a)(x, f(x))=v_{f(x)}(a(x))=h(x)=v_{f(x)}\left(f^{h}(x)\right)=d\left(f^{h}\right)(x, f(x)),
$$

showing that $(d(a)-d(f)) \mid \boldsymbol{D}(f) \cap(U \times \Pi)=0$. We conclude that $\lambda d(a)=\lambda d\left(f^{h}\right)$, and hence that $g=\boldsymbol{h} \boldsymbol{V}(\boldsymbol{f})$, proving the theorem.

THEOREM 6.10. Let $\boldsymbol{M}$ be a maximal ideal of $Z$ that is not principal and let $g_{0}$ be the least positive element of $G$. Then $\boldsymbol{h} \rightarrow \boldsymbol{h} g_{0}$ does not map $\boldsymbol{Z}$ onto $G$,

Proof. We have observed (proof of (6.4)) that there exists $f \in M$ such that $f(x)$ is 1 or is the product of distinct primes, and that $V(f)=g_{0}$. Let $X_{n}=f^{-1}(n)$ for all $n \in N$. Given $x \in X, x \in X_{n}$ for a unique $n \in N$. If $n=1$, let $a(x)=1$. If $n \neq 1$, then $n=p_{1} \cdots \cdots p_{n}, p_{1}, \cdots, p_{n}$ being distinct prime numbers. Let $a(x)=p_{1} \cdot p_{2}^{2} \cdot \cdots \cdot p_{n}^{n}$. Clearly, $a \in C(X, Z)$ and $V(a) \geqq g_{0}$. Assume, for a moment, that there exists $h \in C(X, Z)$ such that $h g_{0}=V(a)$. Since $V(a) \geqq g_{0}, \boldsymbol{h} \geqq 1$ and we can assume, without loss of generality, that $h>0$. Since $V(a)>0$, there exists $m_{0} \in M$ such that $d(a) \mid \boldsymbol{D}\left(m_{0}\right)>0$. Since $\boldsymbol{h} g_{0}=V(a)$, there exists $m_{1} \in M$ such that $(d(a)-h d(f)) \mid \boldsymbol{D}\left(m_{1}\right)=0 . \quad \boldsymbol{D}\left(m_{0}\right) \cap \boldsymbol{D}\left(m_{1}\right)=\boldsymbol{D}\left(\left(m_{0}, m_{1}\right)\right)$, by $[\mathbf{P}$, 2.2.1, (c)]. But $\left(m_{0}, m_{1}\right)=(m)$, where $m \in M[\mathrm{P}, 1.3 .2]$. Since $m \in M, U \equiv \mathscr{U}(m)$ is in $\mu$. By definition, given $x \in U$ there exists $p \in \Pi$ such that $(x, p) \in D(m)$. Since $\boldsymbol{D}(m) \subset \boldsymbol{D}\left(m_{0}\right), d(a)(x, p)>0$ : i.e., $p$ divides $a(x)$. Since $\boldsymbol{D}(m) \subset \boldsymbol{D}\left(m_{1}\right)$, 
$v_{p}(a(x))=h(x) v_{p}(f(x))$. But $a(x)=p_{1} \cdot p_{2}^{2} \cdot \cdots \cdot p_{n}^{n}$ and $f(x)=p_{1} \cdot \cdots \cdot p_{n}$, showing that $p=p_{h(x)}$, where $1 \leqq h(x) \leqq n$ : i.e., $m(x)$ is the prime $p_{h(x)}$. We conclude that $m \mid U$ is a prime-valued function in $C(U, Z)$. Let $m^{\prime}|U=m| U$ and let $m^{\prime} \mid X-U=2$. Then $m^{\prime}$ is a prime-valued function in $C(X, Z), \boldsymbol{m}=\boldsymbol{m}^{\prime}$ and hence, by (5.1), $\boldsymbol{M}$ contains a prime $\boldsymbol{m}$. This shows that $\boldsymbol{M}$ is principal, which is a contradiction, proving the theorem.

THEOREM 6.11. Let $\boldsymbol{M}$ be a maximal ideal of $\boldsymbol{Z}$ and let $g_{0}$ be the least positive element in $\mathrm{G}$. Then $\mathrm{Zg}_{0}$ is cofinal in $\mathrm{G}$.

Proof. If $M$ is principal we may apply (6.9) to obtain the theorem. Assume that $M$ is not principal. There exist $f \in M$ such that $f(x)$ is 1 or is the product of primes. Then $\lambda d(f)=g_{0}$, the least positive element of $G(6.4)$. Let $a \in C(X, Z)$ such that $\lambda d(a) \geqq g_{0}$. There exists $m \in M$ such that $d(a)|\boldsymbol{D}(m) \geqq d(f)| \boldsymbol{D}(m)>0$. Let $U=\mathscr{U}(m)$. For $n, k \in Z$ let $U_{n}=U \cap f^{-1}(n)$ and $V_{k}=U \cap a^{-1}(k)$. Clearly, $\left(U_{n} \cap V_{k}\right)_{(n, k) \in Z \times Z}$ is a partition of $U$ by elements of $\mathscr{B}$. Assume that $U_{n} \cap V_{k} \neq \varnothing$. Then $n=p_{1} \cdot \cdots \cdot p_{j}$ and $k=p_{1}^{e_{1}} \cdot \cdots \cdot p_{j}^{e_{j}}$, where $e_{i}$ is a non-negative integer. Let $h \mid U_{n} \cap V_{k}=\max e_{i}$, and let $h \mid X-U=1$. Then $h \in C(X, Z)$, and $d(a)\left|\boldsymbol{D}((m, f)) \leqq d\left(f^{h}\right)\right| \boldsymbol{D}((m, f))$, proving the theorem.

7. The ideal theory of $Z$. Let $\mathfrak{p}$ be the space of prime ideals of $\boldsymbol{Z}$. $\mathfrak{p}$ is, of course, a compact $T_{0}$-space. Under inclusion $\mathfrak{p}$ is an inductive, partially ordered set, having $\{0\}$ as a least element and having $m$ as the set of maximal elements. Given $\boldsymbol{M} \in \mathfrak{m}$, let $\mathfrak{p}(\subset \boldsymbol{M})=\{\boldsymbol{P} \in \mathfrak{p}: \boldsymbol{P} \subset \boldsymbol{M}\}$.

Given an ideal $I$ in $\boldsymbol{Z}$, let $\boldsymbol{I}_{M}=\boldsymbol{I} \boldsymbol{Z}_{\boldsymbol{M}}$, the extended ideal of $\boldsymbol{I}$ in $\boldsymbol{Z}_{\boldsymbol{M}}$. Let $\boldsymbol{M}$ be a fixed maximal ideal in $Z$, let $V_{M}$ be the valuation of $Q$ associated with $Z_{M}$, and let $G_{M}$ be the value group of $V_{M}$. Clearly, $V_{M}(I)=V_{M}\left(I_{M}\right)$. Since $I_{M}$ is an ideal in $Z_{M}$, $V_{M}\left(I_{M}\right)$ is an upper set in $G_{M} \cup\{\infty\}$. Let $G_{M}(I)$ be $\left\{g \in G_{M}:|g|<V_{M}(f)\right.$, for all $\boldsymbol{f} \in \boldsymbol{I}\}$. Clearly, $G_{M}(I)$ is a convex symmetric subset of $G_{M}$. Let us now apply these notations to the prime ideals in $\boldsymbol{Z}$.

THEOREM 7.1. The mapping $\boldsymbol{P} \rightarrow G_{M}(\boldsymbol{P})$ is a bijective, order-reversing mapping of $\mathfrak{p}(\subset M)$ onto the chain of convex subgroups of $G_{M}$. Thus the order type of $\mathfrak{p}(\subset M)$ is that of the Dedekind completion of an $\eta_{1}$-set with a greatest element adjoined: i.e., $\mathfrak{p}(\subset M)$ is anti-isomorphic to $\bar{S}_{M}$. Finally, given $\boldsymbol{P} \in \mathfrak{p}(\subset M)$ such that $\boldsymbol{P} \neq \mathbf{M}, \boldsymbol{P}$ is not a principal ideal.

Proof. By $\left[\mathrm{ZS}_{1}\right.$, p. 228] and $\left[\mathrm{ZS}_{2}\right.$, p. 40$]$, the first assertion is true. We have shown that the convex subgroups of $G_{M}$ are in bijective, order-preserving correspondence with the lower sets of the value set $S_{M}$ of $G_{M}$, that $S_{M}$ has a least element $s_{0}$, and that $S_{M}-\left\{s_{0}\right\}$ is an $\eta_{1}$-set (6.5), proving the second statement. To prove the last assertion, assume for a moment that there exists a prime ideal $\boldsymbol{P}$ of $Z$, properly contained in $M$, that is principal. Let $P=(f)$; then $V_{M}(f)$ is the least element of $V_{M}(P)$ and $G_{M}(P)$ is a convex subgroup of $G_{M}$. Since $P \neq M, V_{M}(f) \equiv g_{1}$ 
is greater than $g_{0}$, the least positive element of $G_{M}$. Since $G_{M}(P)$ is a subgroup of $G_{M}, W\left(g_{1}\right) \equiv s_{1}>s_{0} \equiv W\left(g_{0}\right)$. By (6.6), $H\left(s_{1}\right)$ is order isomorphic to the reals. Thus there exists $g_{2} \in G_{M}$ such that $0<g_{2}<g_{1}<2 g_{2}$. Hence $g_{2} \in G_{M}(P)$, but $2 g_{2} \notin G_{M}(\boldsymbol{P})$, a contradiction, proving the theorem. (Note: we have not used the full force of (6.6), only the fact that $H\left(s_{1}\right)$ is not discrete.)

Before considering the general ideal theory of $Z$, let us generalize the setting and consider an integral domain $A$, with an identity. Let $m$ be the maximal ideal space of $A$, let $I$ be an ideal in $A$, and let $\mathfrak{m}(I)=\{M \in \mathfrak{m}: I \subset M\} \cdot \mathfrak{m}(I)$, the variety of $I$, is a closed subset of $\mathrm{m}$.

Lemma 7.2. Let $I$ be a proper ideal in $A$. Then $I$ is $\bigcap_{M \in \mathfrak{m}(I)} I_{M} \cap A$.

Proof. Let $J=\bigcap_{M \in \mathfrak{m}(I)} I_{M} \cap A$. If $f \in A-I$, let $B=\{a \in A: a f \in I\}$. Then $B$ is a proper ideal of $A$ containing $I$, and is contained in a maximal ideal $M$. Clearly, $f \notin I_{M}$, so $f \notin J$, showing that $J \subset I$. Since $I \subset J$, the lemma is proved. (I am indebted to the referee for supplying this short proof.)

An ideal $I$ in $A$ will be called primary if it is contained in a unique maximal ideal. (Although this terminology is used in Banach algebra, it is at variance with the use of the term in classical ring theory.) Equivalently, $I$ is primary if $\mathfrak{m}(I)$ consists of one point.

Let us now return to the study of $Z$.

LemMA 7.3. Let $\boldsymbol{M}$ and $\boldsymbol{M}^{\prime}$ be distinct elements of $\mathrm{m}$, the maximal ideal space of $Z$. Given a positive element $g$ of $G_{M}$, there exists $f \in Z$ such that $V_{M}(f)=g$ and $V_{M^{\prime}}(f)=0$.

Proof. Without loss of generality we may assume that $V_{M}=\theta V$ and that $G_{M}=$ ind $\lim _{m \in M} d C(X, Q)^{*} \mid D(m)$ (6.3). Let $a \in C(X, Z)^{*}$ such that $\lambda d(a)=g$. Since $M \neq M^{\prime}$ and since $g>0$, there exist $m \in M$ and $m^{\prime} \in M^{\prime}$ such that $\boldsymbol{D}(m) \cap \boldsymbol{D}\left(m^{\prime}\right)=\varnothing$ and $d(a) \mid \boldsymbol{D}(m)>0$. Let $U=\mathscr{U}(m)$ and let $U^{\prime}=\mathscr{U}\left(m^{\prime}\right)$. Since $P_{\mu}$ is contained in $M$ and in $M^{\prime}, U \cap U^{\prime} \equiv W$ is in $\mu$. Since $D(m) \cap D\left(m^{\prime}\right)=\varnothing$, given $x \in W,\left(m(x), m^{\prime}(x)\right)=(1)$. Let $X_{i}=m^{-1}(i), X_{j}^{\prime}=\left(m^{\prime}\right)^{-1}(j)$ and $U_{k}=a^{-1}(k)$, for all $i, j, k \in Z$. Assume that $X_{i} \cap X_{j}^{\prime} \cap U_{k} \cap W$ is nonempty. Then $(i, j)=(1)$ and $v_{p}(k)>0$ for all prime divisors $p$ of $i$. Let $k= \pm p_{1}^{e_{1}} \cdots \cdot p_{s}^{e_{s}}$, where $p_{1}, \cdots, p_{s}$ are distinct primes, and $e_{1}, \cdots, e_{s} \in N$. Let $p_{1}, \cdots, p_{r}$ be the prime divisors of $k$ that are also prime divisors of $i$. Let $f \mid X_{i} \cap X_{j}^{\prime} \cap U_{k} \cap W=p_{1}^{e_{1}} \cdots \cdots p_{r}^{e_{r}}$ and let $f \mid X-W=1 . D\left(\chi_{X-W}\right)=W \times \Pi$, and $\chi_{X-W} \in M$; thus

$$
\boldsymbol{D}\left(\left(m, \chi_{X-W}\right)\right)=\boldsymbol{D}(m) \cap W \times \Pi,
$$

this set being in $D(M)$. By construction, $(d(f)-d(a)) \mid D\left(\left(m, \chi_{X-W}\right)\right)=0$; thus $\boldsymbol{V}_{\boldsymbol{M}}(\boldsymbol{f})=g$. By construction, $d(f) \mid \boldsymbol{D}\left(\left(m^{\prime}, \chi_{\boldsymbol{X}-W}\right)\right)=0$, showing that $\boldsymbol{V}_{\boldsymbol{M}}(\boldsymbol{f})=0$, proving the lemma.

Using these results together, we get the following. 
THEOREM 7.4. Let $I$ be a nonzero, proper ideal in $Z$, and let $M \in \mathfrak{m}(I)$. $\boldsymbol{I}_{\boldsymbol{M}} \cap \boldsymbol{Z}=\boldsymbol{J}$ is a primary ideal contained in $\boldsymbol{M}$. Further, $\boldsymbol{J}=\left\{\boldsymbol{f} \in \boldsymbol{Z}: \boldsymbol{V}_{\boldsymbol{M}}(\boldsymbol{f}) \in \boldsymbol{V}_{\boldsymbol{M}}(\boldsymbol{I})\right\}$. Finally, $\boldsymbol{I}=\cap_{\boldsymbol{M} \in \mathfrak{m}(I)} \boldsymbol{I}_{M} \cap \boldsymbol{Z}$ is a decomposition of $I$ into primary ideals.

Proof. Since $Z_{M}$ is a valuation $\operatorname{ring}, I_{M}=\left\{f \in Z_{M}: V_{M}(f)>G_{M}\left(I_{M}\right)\right\}\left[\mathrm{ZS}_{2}\right.$, p. 40]. Since $G_{M}(I)=G_{M}\left(I_{M}\right), I_{M}=\left\{f \in Z_{M}: V_{M}(f) \in V_{M}(I)\right\}$. Assume, for a moment, that $\boldsymbol{J}$ is not primary; then it is contained in a maximal ideal $\boldsymbol{M}^{\prime} \neq \boldsymbol{M}$. Since $\boldsymbol{I} \neq(0)$, there exists $g \in V_{M}(I) \cap G_{M}$. By (7.3), there exists $f \in Z$ such that $V_{M}(f)=g$ and $\boldsymbol{V}_{\boldsymbol{M}^{\prime}}(\boldsymbol{f})=0$. Thus, $\boldsymbol{f} \in J$ and $\boldsymbol{f} \notin M^{\prime}$, a contradiction. We conclude that $J$ is primary, proving the theorem.

Applying this theorem now to the special case of primary ideals yields the following.

Corollary 7.5. Let $J$ be a primary ideal of $Z$ contained in $M$. Then, $\boldsymbol{J}_{\boldsymbol{M}} \cap \boldsymbol{Z}=\boldsymbol{J}=\left\{\boldsymbol{f} \in \boldsymbol{Z}: \boldsymbol{V}_{\boldsymbol{M}}(\boldsymbol{f}) \in \boldsymbol{V}_{\boldsymbol{M}}(\boldsymbol{J})\right\}$.

Let $i$ be the set of all primary ideals of $Z$. Under inclusion, $i$ is an inductive partially ordered set whose set of maximal elements is $m$. Given $\boldsymbol{M} \in \boldsymbol{m}$ let $\mathfrak{j}(\subset \boldsymbol{M})=\{\boldsymbol{J} \in \mathrm{j}: \boldsymbol{J} \subset \boldsymbol{M}\}$. Let $\mathfrak{p}^{*}=\mathfrak{p}-\{\boldsymbol{0}\}$ and let $\mathfrak{p}^{*}(\subset \boldsymbol{M})=\mathfrak{p}(\subset \boldsymbol{M}) \cap \mathfrak{p}^{*}$.

THEOREM 7.6. The mapping $\boldsymbol{J} \rightarrow G_{M}(J)$ is a bijective, order-reversing mapping of $\mathrm{j}(\subset M)$ onto the proper, nonempty, symmetric, convex subsets of $G_{M}$. Thus $\mathrm{i}(\subset \mathbf{M})$ is totally ordered under inclusion, and $\mathrm{p}^{*}(\subset \boldsymbol{M})$ is a subset of $\mathrm{j}(\subset \mathbf{M})$. Finally, $\mathrm{j}$ and $\mathfrak{p}^{*}$ are disjoint unions of chains, $\mathrm{i}(\subset \boldsymbol{M})$ and $\mathfrak{p}^{*}(\subset \boldsymbol{M})$; respectively, one for each $M \in \mathrm{m}$.

Proof. By (7.5), $\boldsymbol{J} \rightarrow G_{M}(\boldsymbol{J})$ is an injection of $\mathrm{i}(\subset \boldsymbol{M})$ into the set of nonempty, proper, symmetric, convex subsets of $G_{M}$. Let $G^{\prime}$ be such a subset of $G_{M}$. Then $J^{\prime}=\left\{f \in Z_{M}: V_{M}(f)>G^{\prime}\right\}$ is an ideal in $Z_{M}$. Let $J=J^{\prime} \cap Z$ and note that $G_{M}(J)=G^{\prime}$, proving that the mapping is surjective (7.3). Clearly, these convex subsets of $G_{M}$ form a chain, showing that $\mathrm{i}(\subset M)$ is a chain. We noted before (7.1) that, if $G_{M}(J)$ is a convex subgroup, then $J$ is a prime ideal in $Z$, and conversely, showing that $\mathfrak{p}^{*}(\subset \boldsymbol{M}) \subset \mathrm{j}(\subset \boldsymbol{M})$. By definition, if $\boldsymbol{M}$ and $\boldsymbol{M}^{\prime}$ are distinct maximal ideals, $\mathrm{i}(\subset \boldsymbol{M}) \cap \mathrm{j}\left(\subset \boldsymbol{M}^{\prime}\right)=\varnothing$, proving the last assertion.

Let us turn our attention briefly to the classical radical of an ideal in $Z$.

Proposition 7.7. Let $J \in \mathrm{j}(\subset M)$. Then $J^{1 / 2} \in \mathrm{i}(\subset M)$, and $G_{M}\left(J^{1 / 2}\right)$ is the largest, proper, convex subgroup of $G_{M}(J)$; thus $J^{1 / 2}$ is prime.

Proof. Since $J^{1 / 2}$ contains $J$ and is a proper ideal, it is primary, proving the first assertion. By definition, $V_{M}\left(J^{1 / 2}\right)=\left\{V_{M}(a)\right.$ : there exists $n \in N$ such that $\left.n V_{M}(a) \in V_{M}(J)\right\}$, showing that $V_{M}\left(J^{1 / 2}\right)$ is indeed such a subgroup, proving the proposition.

LEMMA 7.8. Let $I$ be a proper ideal in a commutative integral domain with identity $A$. Then $I^{1 / 2}=\bigcap_{M \in \mathfrak{m}(I)}\left(I_{M} \cap A\right)^{1 / 2}$. 
Proof. Given $f \in I^{1 / 2}$, there exists $n \in N$ such that $f^{n} \in I$. By (7.2), $f^{n} \in I_{M} \cap A$ for all $M \in \mathfrak{m}(I)$; thus $f \in \bigcap_{M \in \mathfrak{m}(I)}\left(I_{M} \cap A\right)^{1 / 2} \equiv L$. If $M \in \mathfrak{m}-\mathfrak{m}(I),\left(I_{M} \cap A\right)^{1 / 2}=A$, showing that $L=\bigcap_{M \in \mathfrak{m}}\left(I_{M} \cap A\right)^{1 / 2}$. Let $f \in L$. Given $M \in \mathfrak{m}$, there exists $n \in N$ such that $f^{n} \in I_{M} \cap A$ : i.e., there exists $a \in I$ and $b \in A-M$ such that $f^{n}=a / b$. Using the fact that $m$ is compact, there exist $b_{1}, \cdots, b_{k} \in A, a_{1}, \cdots, a_{k} \in I$, and $n_{i} \in N$ such that $b_{i} f^{n_{i}}=a_{i}$ for $i=1 m, \cdots, k$ and $\left(b_{1}, \cdots, b_{k}\right)=(1)$. Using the last fact, there exist $c_{1}, \cdots, c_{k} \in A$ such that $c_{1} b_{1}+\cdots+c_{k} b_{k}=1$. We see that $f^{m}=c_{1} b_{1} f^{m}+\cdots+c_{k} b_{k} f^{m} \in I$, for some $m$ in $N$, showing that $f \in I^{1 / 2}$.

Application to general nonstandard arithmetic. Let $A$ be a nonstandard model of $Z$ and let $I$ be a nonzero, proper ideal in $A$. Then $I=\bigcap_{M \in \operatorname{m(I} I} I_{M} \cap A$, and $I_{M} \cap A=\left\{a \in A: V_{M}(a) \in V_{M}(I)\right\}$; however, unless it is known that a result like (7.3) holds for $A$, we cannot conclude that $I_{M} \cap A$ is primary.

In case $\boldsymbol{M}$ is a principal ideal $(\boldsymbol{f})$, we have additional results to utilize. By applying these to ideals $J$ in $\mathrm{i}(\subset(f))$, we obtain the following.

THEOREM 7.9. Let $\boldsymbol{f}$ be a prime element in $Z$ and let $J \in \mathrm{j}(\subset(f))$. Let $g_{0}$ be the least positive element of $G \equiv G_{M(\mu, f)}$. There exists $\left(\boldsymbol{h}_{i}\right)_{i \in I}$, a subset of positive elements of $\boldsymbol{Z}$, such that $\left(\boldsymbol{h}_{i} g_{0}\right)_{i \in I}$ is coinitial in $V_{M}(J)$. Further, $\left(f^{h_{i}}\right)_{i \in I}$ is a set of generators of $J$. Conversely, given any set $\left(\boldsymbol{h}_{i}\right)_{i \in I}$ of positive elements of $Z$, the ideal $\boldsymbol{J}$ generated by $\left(\boldsymbol{f}^{\boldsymbol{h}_{i}}\right)_{i \in I}$ is in $\mathrm{i}(\subset(\boldsymbol{f}))$ and $\left(\boldsymbol{h}_{i} g_{0}\right)_{i \in I}$ is coinitial in $\boldsymbol{V}_{M}(\boldsymbol{J})$.

Proof. By (6.9), such a subset $\left(\boldsymbol{h}_{i}\right)_{i \in I}$ exists. By (6.8) $\boldsymbol{V}_{M}\left(\boldsymbol{f}^{\boldsymbol{h}_{i}}\right)=\boldsymbol{h}_{\boldsymbol{i}} g_{0}$; thus, by (6.7), $\left(f^{h_{i}}\right)_{i \in I}$ is a set of generators of $J_{M}$. Since $J$ is primary, $J_{M} \cap Z=J$ (7.5), proving the first assertion. By construction, $\left(f^{h_{i}}\right)$ is in $\mathrm{j}(\subset(f))$, for each $i \in I$, showing that $J$ is a union of elements in the chain $\mathrm{j}(\subset(f))$, and hence is primary. Since $V_{M}\left(f^{h_{i}}\right)=h_{i} g_{0}$, this set is coinitial in $V_{M}(J)$, proving the theorem.

COROLlaRY 7.10. Let $\boldsymbol{f}$ be a prime element in $Z$. The minimal cardinal number of generating sets of elements of $\mathrm{i}(\subset(f))$ can be $1, \aleph_{0}$, or $\aleph_{1}$.

8. The ideal theory of $C(X, Z)$. The space $\mathfrak{P}$ of prime ideals of $C(X, Z)$ is a compact $T_{0}$-space. As a partially ordered set, $\mathfrak{P}$ is inductive, having $\mathfrak{M}$ as the set of maximal elements and $\mathfrak{P}_{0}$, the space of minimal prime ideals, as the set of minimal elements. The topology on $\mathfrak{M}$ and $\mathfrak{P}_{0}$ is the induced topology. As was seen in $\$ 1$, given $M \in \mathfrak{M}$ there exists a unique minimal prime ideal $\sigma(M)$ contained in $M$. Further, $\sigma$ is a continuous surjection (1.3).

Given $M \in \mathfrak{M}$, let $\mathfrak{P}(\subset M)=\{P \in \mathfrak{P}: P \subset M\}$.

Proposition 8.1. Given $M \in \mathfrak{M}, \mathfrak{P}(\subset M)$ is a chain having a greatest element $M$ and a least element $\sigma(M) \equiv P_{\mu}$. Let $Z=C(X, Z) / \sigma(M)$. $\mathfrak{P}(\subset M)$ is naturally, order isomorphic to $\mathfrak{p}(\subset M)$; thus $\mathfrak{P}(\subset M)$ consists of two points if $\mu \in \delta_{0} X$, and is of power at least $2^{\alpha_{1}}$ if $\mu \notin \delta_{0} X$. Finally, if $\mu \notin \delta_{0} X$, no $P \in \mathfrak{P}(\subset M)$ is principal. 
Most of the proposition follows from the analysis of $\mathfrak{p}(\subset M)$ in $\S 7$. The power of $\mathfrak{P}(\subset M)$ in case $\mu \notin \delta_{0} X$ is computed by using (7.1) and [GJ, 13.24].

Proof. (8.1) implies all but the statement, that if $\mu \notin \delta_{0} X, M$ is not principal. Assume for a moment that $M=(f)$. Since $\mu=\mathscr{U}(M)$ (according to the diagram preceding (1.3)), $\mu=\{U \in \mathscr{B}: \mathscr{U}(f) \subset U\}$ : i.e., $\mathscr{U}(f)$ consists of a single point $x \in X$. This implies that $\mu \in \delta_{0} X$, proving the theorem.

Let $I$ be an ideal in $C(X, Z)$ and let $\mathfrak{M}(I)=\{M \in \mathfrak{M}: I \subset M\}$. Clearly, this is a closed subset of $\mathfrak{M}$. Let $\mathfrak{P}_{0}(I)=\sigma(\mathfrak{M}(I))$. Clearly, an element $P \in \mathfrak{P}_{0}$ is in $\mathfrak{P}_{0}(I)$ if and only if the image of $I$ in $C(X, Z) / P$ is a proper ideal.

Proposition 8.2. $\mathfrak{P}_{0}(I)$ is a closed set in $\mathfrak{P}_{0}$.

Proof. Since $\mathfrak{M}(I)$ is a closed subset of a compact space $\mathfrak{M}$, it is compact. Since $\sigma$ is continuous (1.3), $\mathfrak{P}_{0}(I)$ is compact. Since $\mathfrak{P}_{0}$ is homeomorphic to $\delta X(1.2)$ and since $\delta X$ is Hausdorff $[\mathrm{P}, 1.5 .2], \mathfrak{P}_{0}$ is Hausdorff. We conclude that $\mathfrak{P}_{0}(I)$ is closed, proving the proposition.

An ideal $I$ of $C(X, Z)$ will be called coprimary if $\mathfrak{P}_{0}(I)$ consists of a single point.

Proposition 8.3. Let I be an ideal in $C(X, Z)$. If $P \in \mathfrak{P}_{0}(I)$, then $\mathfrak{P}_{0}(I+P)=\{P\}$ : in other words, $I+P$ is a coprimary ideal.

Proof. Since $P \in \mathfrak{P}_{0}(I)$, there exists $M \in \mathfrak{M}(I)$ such that $\sigma(M)=P$. Clearly $I+P \subset M$, proving that $P \in \mathfrak{P}_{0}(I+P)$. Let $P^{\prime} \in \mathfrak{P}_{0}(I+P)$ and let $M^{\prime} \in \mathfrak{M}(I+P)$ such that $\sigma\left(M^{\prime}\right)=P^{\prime}$. Then $P \subset I+P \subset M^{\prime}$, and $P^{\prime}=P$ by (1.1), proving the proposition.

THEOREM 8.4. Let $I$ be a proper ideal in $C(X, Z)$. Then $I=\bigcap_{P \in \Re_{0}(I)} I+P$ : i.e., $I$ is expressible as the intersection of coprimary ideals $I+P$, one for each $P \in \mathfrak{P}_{0}(I)$.

Proof. Since $I \subset I+P$ for all $P \in \mathfrak{P}_{0}, I \subset \bigcap_{P \in \mathfrak{P}_{0}(I)} I+P \equiv J$. If $P \in \mathfrak{P}_{0}-\mathfrak{P}_{0}(I)$, then the image of $I$ in $C(X, Z) / P$ is contained in no maximal ideal, showing that $I+P=C(X, Z)$; thus $J=\bigcap_{P \in \Re_{0}} I+P$. Let $f \in J$. It suffices to show that $f \in I$. By definition, given $P \in \mathfrak{P}_{0}$, there exists $g \in I$, depending on $P$, such that $f-g \in P$. By (1.4), $\mathfrak{P}_{0}(f-g)$ is an open set in $\mathfrak{P}_{0}$. Since $\mathfrak{P}_{0}$ is compact, there exist $g_{1}, \cdots, g_{n} \in I$ such that $\mathfrak{P}_{0}\left(f-g_{1}\right), \cdots, \mathfrak{P}_{0}\left(f-g_{n}\right)$ cover $\mathfrak{P}_{0}$. As a consequence, $U_{1} \equiv \zeta\left(f-g_{1}\right), \cdots, U_{n} \equiv \zeta\left(f-g_{n}\right)$ constitute a cover of $X$ by elements of $\mathscr{B}$. Let $V_{1}=U_{1}, V_{2}=U_{2}-V_{1}$, and $V_{3}=U_{3}-\left(V_{1} \cup V_{2}\right)$. Proceeding in this fashion, by finite induction, one can define a partition $V_{1}, \cdots, V_{n}$ of $X$ by elements of $\mathscr{B}$ that is a refinement of $U_{1}, \cdots, U_{n}$. Let $g=g_{1} \chi_{V_{1}}+\cdots+g_{n} \chi_{V_{n}}$. Since $g_{i} \in I$ and $V_{i} \in \mathscr{B}, g \in I$. Given $x \in X$ there exists a unique $i, 1 \leqq i \leqq n$, such that $x \in V_{i}$. We know that $V_{\imath} \subset U_{i}=\zeta\left(f-g_{i}\right)$, thus $f(x)=g_{i}(x)$. Since $i$ is unique, $g(x)=g_{i}(x)$, showing that $f=g$. Since $g \in I$, we have shown that $f \in I$, proving the theorem. 
THeOREM 8.5. Let I be a proper ideal in $C(X, Z)$. Then $I^{1 / 2}=\bigcap_{P \in \Re_{0}(I)}(I+P)^{1 / 2}$. Further, $(I+P)^{1 / 2}$ is a coprimary ideal.

Proof. Given $f \in I^{1 / 2}$, by definition there exists $n \in N$ such that $f^{n} \in I$. By I(8.4), $f^{n} \in I+P$ for all $P \in \mathfrak{P}_{0}(I)$; thus $f \in \bigcap_{P \in \mathfrak{B}_{0}(I)}(I+P)^{1 / 2} \equiv L$. Conversely, let $f \in L$. Clearly, $L=\bigcap_{P \in \mathfrak{P}_{0}}(I+P)^{1 / 2}$; thus, given $P \in \mathfrak{P}_{0}$, there exist $n \in N$ and $g \in I$ such that $f^{n}-g \in P$. By (1.4), $\mathfrak{P}_{0}\left(f^{n}-g\right)$ is an open set in $\mathfrak{P}_{0}$. Clearly, sets of this form constitute a cover of $\mathfrak{P}_{0}$. Since $\mathfrak{P}_{0}$ is compact, there exist $g_{1}, \cdots, g_{k} \in I$ and $n \in N$ such that $\mathfrak{P}_{0}\left(f^{n}-g_{1}\right), \cdots, \mathfrak{P}_{0}\left(f^{n}-g_{k}\right)$ cover $\mathfrak{P}_{0}$. Let $U_{i} \equiv \zeta\left(f^{n}-g_{i}\right), i=1, \cdots, k$. Clearly, $\left(U_{i}\right)$ is a cover of $X$ composed of elements of $\mathscr{B}$. Using the method described in the proof of (8.4), we can choose a refinement $\left(V_{i}\right)$ of $\left(U_{i}\right)$ that partitions $X$ and is made up of elements of $\mathscr{B}$. Let $g=g_{1} \chi_{V_{1}}+\cdots+g_{k} \chi_{V_{k}}$. Then $g \in I$, and $g=f^{n}$, showing that $f \in I^{1 / 2}$, completing the proof.

Let $I$ be a coprimary ideal and let $\mathfrak{P}_{0}(I)=\{P\}$. Let $Z=C(X, Z) / P$ and let $I$ be the image of $I$ in $Z$. By (7.4), $I=\bigcap_{M \in \mathfrak{m}(I)} I_{M} \cap Z, I_{M} \cap Z$ being a primary ideal in $Z$. Since $I$ is coprimary, $I$ is the full pre-image of $I$, in $C(X, Z)$. The preimage of $\mathfrak{m}(I)$ in $\mathfrak{M}$ is $\mathfrak{M}(I)$. Further, the pre-image of $I_{M} \cap Z$ is primary and is $\{f \in C(X, Z): b f=a \in I$ for some $b \in C(X, Z)-M\} \equiv I_{M} \cap C(X, Z)$. Finally, $I^{1 / 2}=\bigcap_{M \in \mathfrak{m}(I)}\left(I_{M} \cap Z\right)^{1 / 2}(7.8)$.

THEOREM 8.6. Let I be a coprimary ideal in $C(X, Z)$. Then the following decompositions hold: $I=\bigcap_{M \in \mathfrak{M}(I)}\left(I_{M} \cap C(X, Z)\right)$, and $I^{1 / 2}=\bigcap_{M \in \mathfrak{M}(I)}\left(I_{M} \cap C(X, Z)\right)^{1 / 2}$, the ideals $\left(I_{M} \cap C(X, Z)\right)^{1 / 2}$ being prime.

Combining (8.4), (8.5), and (8.6), one obtains the following.

THEOREM 8.7. Let I be a proper ideal in $C(X, Z)$. The following decompositions hold: $I=\bigcap_{P \in \mathfrak{B}_{0}(I)} \bigcap_{M \in \mathfrak{M}(I+P)}\left(I_{M} \cap C(X, Z)\right)$, and

$$
I^{1 / 2}=\bigcap_{P \in \Re_{0}(I)} \bigcap_{M \in \mathfrak{W}(I+P)}\left(I_{M} \cap C(X, Z)\right)^{1 / 2} .
$$

LEMMA 8.8. Let $I$ be a proper ideal in $C(X, Z) . \mathfrak{M}(I)=\bigcup_{P \in \mathfrak{P}_{0}(I)} \mathfrak{M}(I+P)$, and given distinct points $P$ and $P^{\prime}$ in $\mathfrak{P}_{0}(I), \mathfrak{M}(I+P) \cap \mathfrak{M}\left(I+P^{\prime}\right)=\varnothing$.

Proof. Let $M \in \mathfrak{M}(I)$. Let $P=\sigma(M)$; then $M \in \mathfrak{M}(I+P)$ and $P \in \mathfrak{P}_{0}(I)$. Conversely, let $M \in \mathfrak{M}(I+P)$ for some $P \in \mathfrak{P}_{0}(I)$. Then $I \subset I+P \subset M$, and $M \in \mathfrak{M}(I)$, proving the first assertion. Let $P, P^{\prime} \in \mathfrak{P}_{0}$ and let $M \in \mathfrak{M}(I+P) \cap \mathfrak{M}\left(I+P^{\prime}\right)$. Since $P$ and $P^{\prime}$ are contained in $M$, we may apply (1.1) and conclude that $P=P^{\prime}$, proving the lemma.

Applying (8.8) to (8.7), we obtain the following.

COROLlaRY 8.9. Let I be a proper ideal in $C(X, Z)$. Then the following deco mpositions of I in terms of primary ideals, and prime ideals respectively, hold: $I=\bigcap_{M \in \mathfrak{M}(I)}\left(I_{M} \cap C(X, Z)\right)$ and $I^{1 / 2}=\bigcap_{M \in \mathfrak{M}(I)}\left(I_{M} \cap C(X, Z)\right)^{1 / 2}$. 
With the aid of this decomposition theorem we can now analyze the set $\mathfrak{I}$ of primary ideals of $C(X, Z)$. Given $J \in \mathfrak{I}, J=J_{M} \cap C(X, Z)$, where $\{M\}=\mathfrak{M}(J)$ (8.9). If $P$ and $P^{\prime}$ are in $\mathfrak{P}_{0}(J)$, then $P=\sigma(M)=P^{\prime}(1.1)$, showing that $J$ is coprimary. Given $P \in \mathfrak{P}_{0}$, let $\mathfrak{I}(\supset P)=\{J \in \mathfrak{I}: J \supset P\}$. Since each primary ideal is coprimary, $\mathfrak{I}$ is a disjoint union of $\mathfrak{I}(\supset P)$, where $P$ runs over $\mathfrak{P}_{0}$. Let $P \in \mathfrak{P}_{0}$ and let $Z=C(X, Z) / P$. The mapping $J \rightarrow J$ of $\mathfrak{I}(\supset P)$ into the ideals of $Z$ is a bijection onto $\mathfrak{j}$, the set of primary ideals of $\boldsymbol{Z}$. By (7.6), $\mathfrak{j}$ is a disjoint union of chains $\mathfrak{i}(\subset M)$. Given $M \in \mathfrak{M}$, let $\mathfrak{I}(\subset M)=\{J \in \mathfrak{J}: J \subset M\}$. Summarizing, we have the following.

THEOREM 8.10. The set $\mathfrak{I}$ of primary ideals of $C(X, Z)$ is a disjoint union of chains $\mathfrak{I}(\subset M)$, one for each $M \in \mathfrak{M}$. $\mathfrak{I}(\subset M)$ is naturally order isomorphic to $\mathrm{j}(\subset M)$, the set of primary ideals in $C(X, Z) / \sigma(M)$ contained in $M$.

Let $I$ be a proper ideal in $C(X, Z)$. By $[\mathrm{P}, 2.3 .4], D(I)$ is a proper filter in $\mathscr{D}$ $(=D C(X, Z))$. By $[\mathrm{P}, 2.3 .4], D^{-1} D(I)$ is a proper ideal in $C(X, Z)$. Clearly it contains $I$. $I$ will be called a $D$-ideal if $I=D^{-1} D(I)$. (Cf. the notion of a $z$-ideal in [GJ].) By $[\mathrm{P}, 2.5 .1]$, a proper $D$-ideal $I$ is $\bigcap_{M \in \mathfrak{M}(I)} M$. If $I$ is now merely a proper ideal of $C(X, Z)$, then $J=D^{-1} D(I)$ is the smallest $D$-ideal that contains $I$. By (8.9), $I=\bigcap_{M \in \mathfrak{M}(I)}\left(I_{M} \cap C(X, Z)\right)$, and $J=\bigcap_{M \in \mathfrak{M}(J)} M$. Since $D(J)=D(I)$, $\mathfrak{M}(I)=\mathfrak{M}(J)$.

Using these results we can obtain additional information about the ideal $I=\bigcap_{p \in \Pi}(p)$ in $Z=C(X, Z) / P_{\mu}$, where $\mu \in \delta X-\delta_{0} X$. Let $I$ be the pre-image of $I$ in $C(X, Z)$. Clearly, $f \in I$ if and only if for all $p \in \Pi$ there exists $U \in \mu$ such that $f(x) \equiv 0(p)$ for all $x \in U$ : i.e., for all $p \in \Pi$ there exists $U \in \mu$ such that $U \times\{p\} \subset D(f)$. Since this is a condition on $D(f), I$ is a $D$-ideal and is, by $[P, 2.5 .1$.$] , an intersection of maximal ideals. We conclude that I$ is the inter-

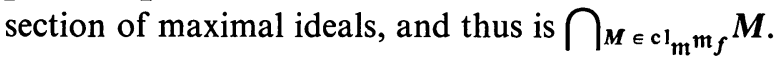

9. Some remarks on the residue class fields of $C(X, Z)$. Pierce has obtained a great deal of information about the residue class fields of $C(X, Z)$. One of his most striking results deals with the elementary theory of these fields [P, 6.4.1]. Before this theorem is stated, consider the following background material. Let $\pi$ be the projection mapping of $X \times \Pi$ onto $\Pi$. Given $f \in C(X, Z)$, let $\mathscr{P}(f)=\pi \boldsymbol{D}(f)$. Given an ideal $I$ in $C(X, Z)$, let $\mathscr{P}(I)=\{\Delta \subset \Pi: \mathscr{P}(f) \subset \Delta$ for some $f \in I\}$. Clearly, $\mathscr{P}(I)$ is a filter on $\Pi$. Assume that $X$ is not $Z$-pseudocompact; then $X$ has an infinite partition $\left(U_{n}\right)_{n \in N}$ made up of elements of $\mathscr{B}$. Let $\Delta \in \mathscr{P}(I)$ and let $f \in I$ such that $\mathscr{P}(f) \subset \Delta$. Let $\Delta=\left(p_{n}\right)_{n \in N}$ and let $g \mid U_{n}=p_{1} \cdots \cdots p_{n}$. Then $\mathscr{P}(g)=\Delta$, $f g \in I$, and $\mathscr{P}(f g)=\Delta$, showing that $\mathscr{P}(I)=\pi D(I)$ in case $X$ is not Z-pseudocompact. It has been shown that, if $M \in \mathfrak{M}$, then $\mathscr{P}(M)$ is in $\beta \Pi$, the space of ultrafilters on $\Pi[\mathrm{P}, 4.1 .4]$. Let $\phi \in L(\mathfrak{U})$ (see $\S 3$ for definitions), where $\mathfrak{R}$ is the category of all commutative rings aud ring homomorphisms, let $\Pi(\phi)=\{p \in \Pi: \phi$ is valid in $R /(p)\}$. 
TheORem 9.1 (PierCe $[\mathrm{P}, 6.4 .1]$ ). Let $M \in \mathfrak{M}$ and let $\phi \in L(\mathfrak{R}) . \phi$ is valid in $C(X, Z) / M$ if and only if $\Pi(\phi) \in \mathscr{P}(M)$.

Thus, the elementary theory of $C(X, Z) / M$ is determined by $\mathscr{P}(M)$. Let $p \in \Pi$ be identified with the fixed ultrafilter at $p$. $\Pi$ is imbedded as a discrete, dense subspace of $\beta \Pi$. If $\mathscr{P}(M)=p$, we may use $[\mathrm{P}, 3.3 .3]$ and conclude that $C(X, Z) / M$ is isomorphic to $Z /(p)$, and conversely (see also (4.2)). By [P, 4.2.2], $\mathscr{P}$ maps $\mathfrak{M}$ onto $\beta \Pi$, if $X$ is not $Z$-pseudocompact.

Let $\mu \in \delta X, Z=C(X, Z) / P_{\mu}$, and let $M \in \mathscr{U}^{-1}(\mu)$. Clearly, the mapping $C(X, Z) \rightarrow C(X, Z) / M$ factors through $Z$ and $C(X, Z) / M$ is isomorphic to $Z / M$. Thus the choice of $\mu$ has a decisive effect on the residue class fields that can arise.

Proposition 9.2. Let $\mu \in \delta_{0} X$. Then $\mathscr{P}$ maps $\mathscr{U}^{-1}(\mu)$ onto $\Pi$, Let $\Pi$ be topologized by letting the finite subsets be a basis of closed sets; then $\mathscr{P}$ is a homeomorphism of $\mathscr{U}^{-1}(\mu)$ onto $\Pi$. Conversely, let $\mu \in \delta X$ and assume that $\mathscr{P}$ maps $\mathscr{U}^{-1}(\mu)$ into $\Pi$; then $\mu \in \delta_{0} X$.

Proof. Let $\mu \in \delta_{0} X$ and let $\boldsymbol{Z} \equiv C(X, Z) / P_{\mu}$. By definition, $\boldsymbol{Z}$ is isomorphic to $Z$. By (2.2), $M \rightarrow M$ is a homeomorphism of $\mathscr{U}^{-1}(\mu)$ onto $\mathfrak{m}$. Each $\boldsymbol{M} \in \mathfrak{m}$ is of the form (p) for some $p \in \Pi$; thus $M=M(p, \mu)(5,4)$, and $\mathscr{P}(M)=p$, proving the first assertion. Conversely, assume that $\mu \in \delta X$ such that $\mathscr{P}$ maps $\mathscr{U}^{-1}(\mu)$ into $\Pi$. Assume for a moment that $Z \equiv C(X, Z) / P_{\mu}$ is not isomorphic to $Z$. By (5.3), there exists an infinite prime $\boldsymbol{f}$ in $\boldsymbol{Z}$. By (5.1), $\boldsymbol{f}$ has a prime-valued pre-image $f$ in $C(X, Z)$. By (5.4), $M(f, \mu) \rightarrow(f)$. Since $f$ is an infinite prime in $Z, \mathscr{P}(M(f, \mu)) \notin \Pi$ $[\mathrm{P}, 3.3 .3]$, a contradiction, showing that $\boldsymbol{Z}=Z$, and proving the proposition.

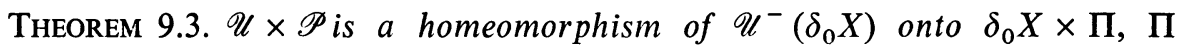
having the topology determined by letting the finite sets of $\Pi$ be basic closed sets.

Proof. By (9.2), $\mathscr{U} \times \mathscr{P}$ is an injective map of $\mathscr{U}^{-1}\left(\delta_{0} X\right)$ onto $\delta_{0} X \times \Pi$. The map $\mathscr{U} \times \mathscr{P}$ composed with both the first and second projection of $\delta_{0} X \times \Pi$ is continuous, by (1.3) and (9.2); thus $\mathscr{U} \times \mathscr{P}$ is continuous. (Since $\delta_{0} X \times \Pi$ is not a $T_{2}$-space we can not use the usual argument to prove that $\mathscr{U} \times \mathscr{P}$ is a homeomorphism.) By (2.4), $C(X, Z)$ and $C\left(\delta_{0} X, Z\right)$ are naturally isomorphic. Thus we may assume that $X=\delta_{0} X$, without loss of generality. $(\mathfrak{M}(f))_{f \in C(X, Z)}$ is a basis of the closed sets of $\mathfrak{M}$; thus it suffices to prove that $(\mathscr{U} \times \mathscr{P})\left(\mathfrak{M}(f) \cap \mathscr{U}^{-1}(X)\right)$ is closed in $X \times \Pi$. Using (5.4) it is easy to see that this set is $\boldsymbol{D}(f)$. To show that $\boldsymbol{D}(f)$ is closed in $X \times \Pi$ note that $X_{n} \equiv f^{-1}(n)$ is in $\mathscr{B}$ for all $n \in Z$; thus $T_{n} \equiv\{(x, p) \in X \times \Pi$ : $x \in X_{n}$ implies $\left.n \equiv 0(p)\right\}$ is closed in $X \times \Pi$. Since $D(f)=\bigcap_{n \in Z} T_{n}$, the theorem is proved.

Given $\mu \in \delta X-\delta_{0} X$, we will consider the map $\mathscr{P}$ of $\mathscr{U}^{-1}(\mu)$ into $\beta \Pi$; but first some technical results.

Lemma 9.4. Let $\mu \in \delta X-\delta_{0} X$, let $\rho \in \beta \Pi$, and let $\Delta_{0} \in \rho$. There exists $f \in C(X, Z)$ such that $D(f)=\Delta_{0}$, and such that $\mathscr{P}\left(\left(f, \chi_{X-v}\right)\right) \in \rho$ for all $U \in \mu$. 
Proof. Since $\mu \notin \delta_{0} X$, there exists a distinguished partition $\left(V_{n}\right)_{n \in N}$ of $X$ relative to $\mu$ (5.2). Let $\Delta_{0}=\left(p_{n}\right)_{n \in N}$ and let $f \mid V_{n}=p_{1} \cdot \cdots \cdot p_{n}$. Then $f \in C(X, Z)$ and $\mathscr{P}(f)=\Delta_{0}$. Let $U_{m}=\bigcup_{n \geqq m} V_{n}$; then, by the definition of $\left(V_{n}\right)_{n \in N}, U_{m} \in \mu$. Let $U \in \mu$ and $\Delta \in \rho$. Since $\rho$ is a filter, there exists $p_{m} \in \Delta \cap \Delta_{0}$. Since $\mu$ is a filter, $U_{m} \cap U \neq \varnothing$. Let $x$ be a point in this set; then $x \in V_{n}$ for some $n \geqq m$. Since $f(x)=p_{1} \cdot \cdots \cdot p_{n},\left(x, p_{m}\right) \in D(f)$. Hence $\mathscr{P}\left(\left(f, \chi_{x-U}\right)\right) \cap \Delta \neq \varnothing$ for all $\Delta \in \rho$. Since $\rho$ is an ultrafilter, the result obtained above shows that $\mathscr{P}\left(\left(f, \chi_{X-U}\right)\right) \in \rho$, proving the lemma.

Given $\mu \in \delta X$ and $\rho \in \beta \Pi$, let $J(\mu, \rho)=\left\{f \in C(X, Z): \mathscr{P}\left(\left(f, \chi_{X-U}\right)\right) \in \rho\right.$ for all $U \in \mu\}$. Clearly, $J(\mu, \rho)$ is a proper $D$-ideal.

Corollary 9.5. Let $\mu \in \delta X-\delta_{0} X$ and let $\rho \in \beta \Pi$. Then $P_{\mu} \subset J(\mu, \rho)$ and $\mathscr{P}(J(\mu, \rho))=\rho$.

Proof. If $f \in P_{\mu}$, then $\mathscr{P}\left(\left(f, \chi_{x}-v\right)\right)=\Pi$ for all $U \in \mu$, showing that $f \in J(\mu, \rho)$. Let $\Delta_{0} \in \rho$ and let $f$ be a function satisfying the conditions of (9.4). Then $f \in J(\mu, \rho)$ and $\mathscr{P}(f)=\Delta_{0}$, proving the corollary.

THEOREM 9.6. Let $\mu \in \delta X-\delta_{0} X$. Then $\mathscr{P}$ maps $\mathscr{U}^{-1}(\mu)$ onto $\beta \Pi$.

Proof. Let $\rho \in \beta \Pi$ and let $M$ be a maximal ideal that contains $J(\mu, \rho)$. By (9.5), $P_{\mu} \subset M$; thus $\mathscr{U}(M)=\mu$. Again using (9.5), $\rho=\mathscr{P}(J(\mu, \rho)) \subset \mathscr{P}(M)$, proving the theorem.

Combining $[\mathrm{P}, 6.4 .1](=(9.1))$ and $(9.6)$, we obtain the following.

THEOREM 9.7, Let $X$ and $Y$ be nonempty Hausdorff spaces, let $K$ be any residue class field of $C(Y, Z)$, let $\mu \in \delta X-\delta_{0} X$, and let $Z=C(X, Z) / P_{\mu}$. There exists a maximal ideal $M$ in $Z$ such that $K$ and $Z / M$ are elementarily equivalent.

Proposition 9.8. Let $M \in \mathfrak{M}$ such that $\mu=\mathscr{U}(M)$. Then $p=\mathscr{P}(M)$ if and only if $M=M(p, \mu)$.

Proof. If $p=\mathscr{P}(M)$, then there exists $m \in M$ such that $\boldsymbol{D}(m)=X \times\{p\}$. This implies that $p \in M, p \in M$, and, by (5.4), that $M=M(p, \mu)$. If $M=M(p, \mu)$, then $p \in \mathscr{P}(M)$, proving the proposition.

Pierce has raised several questions concerning these residue class fields [P, §7]. One $[\mathrm{P}, 7.2]$ asks about relations between $\mathscr{P}(M)$ and $\mathscr{U}(M)$. (9.2), (9.6), and (9.8) effectively answer this question. Pierce goes on to inquire $[\mathrm{P}, 7.3]$ whether two maximal ideals $M$ and $M^{\prime}$ of $C(X, Z)$ are equal if $\mathscr{P}(M)=\mathscr{P}\left(M^{\prime}\right)$ and $\mathscr{U}(M)$ $\mathscr{U}\left(M^{\prime}\right)$. If $\mathscr{U}(M) \equiv \mu \in \delta_{0} X$, then $\mathscr{P}(M)=p$ and $M=M(\mu, p)=M^{\prime}$ (9.8). In (5.9) we saw that $X$ and $\mu$ could be chosen so that $\mathrm{m}_{p}$ exceeds any preassigned cardinal number. Thus $\mathscr{U}^{-1}(\mu)$ can have power greater than that of $\beta \Pi$, and $\mathscr{P} \mid \mathscr{U}^{-1}(\mu)$ cannot be injective in this case, showing that $M$ need not equal $M^{\prime}$, settling $[\mathrm{P}, 7.3]$.

By $[\mathrm{P}, 6.4 .1](=(9.1))$ and $[\mathrm{K}, 5.1]$, if we let $\rho$ run through $\beta \Pi$ and let $Z /(\rho) \equiv \operatorname{ind}_{\Delta \in \rho} \Pi_{p \in \Pi} Z /(p) \mid \Delta$, then these fields form a set of representatives 
of the equivalence classes of elementarily equivalent residue class fields of $C(X, Z)$, in case $X$ is not $Z$-pseudocompact. The power of $\beta \Pi$ is $2^{c}$, where $c$ is the power of the continuum. That this is a very redundant set of representatives can be seen by applying the Löwenheim-Skolem theorem $[R, 1.5 .13]$ and choosing a countable field $K_{\rho}$ for each $\rho \in \beta \Pi-\Pi$ that is elementarily equivalent to $Z /(\rho)$. For $\rho=p$, let $K_{\rho}=Z /(p)$. By (9.8), $\rho \notin \Pi$ implies $K_{\rho}$ is of characteristic zero. Let $\Omega$ be an algebraically closed field of characteristic zero whose transcendence degree over $Q$ is $\aleph_{0}$. Thus $\Omega$ is countable and each $K_{\rho}$ can be imbedded in $\Omega$, $\rho \notin \Pi$. We conclude that there are at most $c$ equivalence classes of elementarily equivalent residue class fields of $C(X, Z)$, and that the elementary theory of $C(X, Z) / M$ does not determine $\mathscr{P}(M)$.

\section{REFERENCES}

[A $\mathrm{A}_{1}$ ] N. L. Alling, A characterization of Abelian $\eta_{\alpha}$-groups in terms of their natural valuation, Proc. Nat. Acad. Sci. U.S.A. 47 (1961), 711-713.

$\left[\mathrm{A}_{2}\right]-$ On the existence of real-closed fields that are $\eta_{\alpha}$-sets of power $\aleph_{\alpha}$, Trans. Amer. Math. Soc. 103 (1962), 341-352.

$\left[\mathrm{A}_{3}\right]-$, An application of valuation theory to rings of continuous real and complexvalued functions, Trans. Amer. Math. Soc. 109 (1963), 492-508.

[A $]$ - The valuation theory of meromorphic function fields over open Riemann surfaces, Acta Math. 110 (1963), 79-96.

[B] G. Birkhoff, Lattice theory, Amer. Math. Soc. Colloq. Publ. Vol. 25, Amer. Math. Soc., Providence, R. I., 1960.

[ES] S. Eilenberg and N. Steenrod, Foundations of algebraic topology, Princeton Univ. Press, Princeton, N. J., 1952.

[GJ] L. Gillman and M. Jerison, Rings of continuous functions, Van Nostrand, Princeton, N. J., 1960.

[H $\mathrm{H}_{1}$ F. Hausdorff, Grundzüge der Mengenlehre (Leipzig, 1914), Chelsea, New York, 1949.

$\left[\mathrm{H}_{2}\right]$ L. Hewitt, Rings of real-valued continuous functions. I, Trans. Amer. Math. Soc. 64, (1948), 45-99.

[K] S. Kochen, Ultraproducts in the theory of models, Ann. of Math. (2) 74 (1961), 221-261.

[P] R. S. Pierce, Rings of integer-valued continuous functions, Trans. Amer. Math. Soc. 100 (1961), 371-394.

[R] A. Robinson, Introduction to model theory and the metamathematics of algebra, NorthHolland, Amsterdam, 1963.

[S] D. Scott, On constructing models for arithmetic, Infinistic methods, Internat. Math. Union, Warsaw, 1961.

[ZS $\mathrm{ZS}_{1}$ R. Zariski and P. Samuel, Commutative algebra. I, Van Nostrand, Princeton, N. J. 1958.

[ZS 2$] \longrightarrow$, Commutative algebra. II, Van Nostrand, Princeton, N.J., 1960.

Massachusetts Institute of Technology,

Cambridge, Massachusetts

PURDUE UNIVERSITY,

LAFAYETTE, INDIANA 